\title{
Insights into Aldehyde Dehydrogenase Enzymes: A Structural Perspective
}

\author{
Kim Shortall, Ahmed Djeghader, Edmond Magner and Tewfik Soulimane* \\ Department of Chemical Sciences, Bernal Institute, University of Limerick, Limerick, Ireland
}

Aldehyde dehydrogenases engage in many cellular functions, however their dysfunction resulting in accumulation of their substrates can be cytotoxic. ALDHs are responsible for the $\mathrm{NAD}(\mathrm{P})$-dependent oxidation of aldehydes to carboxylic acids, participating in detoxification, biosynthesis, antioxidant and regulatory functions. Severe diseases, including alcohol intolerance, cancer, cardiovascular and neurological diseases, were linked to dysfunctional ALDH enzymes, relating back to key enzyme structure. An in-depth understanding of the ALDH structure-function relationship and mechanism of action is key to the understanding of associated diseases. Principal structural features 1) cofactor binding domain, 2) active site and 3) oligomerization mechanism proved critical in maintaining ALDH normal activity. Emerging research based on the combination of structural, functional and biophysical studies of bacterial and eukaryotic ALDHs contributed to the appreciation of diversity within the superfamily. Herewith, we discuss these studies and provide our interpretation for a global understanding of ALDH structure and its purpose-including correct function and role in disease. Our analysis provides a synopsis of a common structure-function relationship to bridge the gap between the highly studied human ALDHs and lesser so prokaryotic models.

Keywords: aldehyde dehydrogenase, NAD(P) cofactor, structure-function, mutations, enzyme dysfunction, oligomerization, C-terminal extensions, spirosomes

\section{INTRODUCTION}

Aldehyde dehydrogenases (ALDH) (EC 1.2.1.3) are a family of nicotinamide adenine dinucleotide (phosphate) (NAD(P)) dependent enzymes, typically with a molecular mass of ca. 50-60 kDa. They oxidise a large range of aliphatic and aromatic, endogenous and exogenous aldehydes to form the corresponding carboxylic acids. They differ in their subcellular location, tissue distribution and preferred substrates while contributing to a broad spectrum of associated biological activities across prokaryotes, eukaryotes and Archaea. ALDHs are involved in detoxification, biosynthesis, antioxidant functions and structural and regulatory mechanisms (Vasiliou and Nebert, 2005). Interestingly, some if not most, ALDHs have multiple functionality. For example, human ALDH1A1, ALDH2, ALDH3A1, and ALDH4A1 can carry out esterase activity Sládek (2003), with ALDH2 also

Received: 27 January 2021 Accepted: 28 April 2021

Published: 14 May 2021

Citation:

Shortall K, Djeghader A, Magner E and Soulimane $T$ (2021) Insights into Aldehyde Dehydrogenase Enzymes: A

Structural Perspective.

Front. Mol. Biosci. 8:659550.

doi: $10.3389 /$ fmolb.2021.659550

Abbreviations: ADH, Alcohol dehydrogenase; AdhE, Alcohol-aldehyde dehydrogenase; ALDH, Aldehyde dehydrogenase; FAD, Flavin adenine dinucleotide; FALDH, Fatty aldehyde dehydrogenase; LsALDH16, ALDH16 from Loktanella Sp.; NAD(P), Nicotinamide adenine dinucleotide (phosphate); PDE, pyridoxine-related epilepsy; PpALDH, ALDH from Pseudomonas putida; SaALDH, ALDH from Staphlococcus aureus; ScALDH4A1, ALDH4A1 from Saccharomyces cerevisiae; SEC, Substrate entry channel; TtALDH 530 , ALDH530 from Thermus thermophilus; TtP5CDH, Pyrroline-5-carboxylate dehydrogenase from Thermus thermophilus 
possessing nitrate reductase activity Sydow et al. (2004), suggesting more than one catalytic function of the ALDH family.

Unlike many other systems, ALDH investigation originated in human models, rather than bacterial ones which likely arises from their central link with pathological conditions. To date 10 out of the 19 human ALDHs have a resolved structure. Initially this led to misconceptions and a rather inaccurate description of typical features related to the enzyme family (Rodríguez-Zavala et al., 2006; Hayes et al., 2018). Emerging research allows for an accurate understanding of diversity within the ALDH family, across prokaryotes and eukaryotes, while bridging the gap to form a global perception of the superfamily. In addition, new enzyme structures demonstrating novel characteristics, provides scope for an updated, complete summary of the ALDH family structure to date.

ALDHs are generally grouped according to sequence identity, phylogeny and structural features combined, with the system evolving through identification of new superfamily members over time. Initial classification of ALDHs commenced in the late 1980s when only few ALDH sequences were available and which led to their classification into 3 distinct classes: ALDH 1, 2 and 3 Lindahl and Hempel (1990), Lindahl (1992), generally composed of human or mammalian isozymes. Classes 1 and 3 consist of both constitutively expressed and inducible cytosolic enzymes, with class 3 being expressed specifically in tumor, stomach and corneal cells. Class 2, contains constitutive mitochondrial enzymes. Each class oxidises a variety of substrates that can be derived from either an endogenous or exogenic source, including aldehydes derived from xenobiotic metabolism (Lindahl, 1992). Class 1 and 2 ALDHs contain mostly substrate variable enzymes while class 3 contains substrate specific ones (Perozich et al., 1999b). Generalisation with regards to the different classes has proposed that class 1 and 2 members, usually homotetramers, contain 500 amino acids per monomer with $70 \%$ sequence identity. Class 3 members, on the other hand, contain 450 amino acids, harboring an $\mathrm{N}$-terminal deletion, and are of homodimeric nature (Rodríguez-Zavala et al., 2006). The sequence identity of class 3 in relation to class 1 or class 2 is only $25 \%$ (Perozich et al., 1999a). With a growing number of ALDH sequences, a new classification based on Dayhoff's work Dayhoff (1976) was adopted later in 1999 to establish rules for ALDH nomenclature. Proteins with sequence identity greater than $40 \%$ were considered to belong to the same class, while proteins with more than $60 \%$ sequence identity have been assigned to the same sub-class (Vasiliou et al., 1999). ALDH nomenclature now spans from ALDH1 to ALDH24 across organisms (Brocker et al., 2013; Hou and Bartels, 2015). Supporting this, the human ALDH family consists of 19 putatively functional genes encoded on distinct chromosomal locations. However, emerging research demonstrates that significantly more ALDH classes exist. A recent study on the Pseudomonas genus identified 42 different classes of ALDHs demonstrating the scope of this enzyme's diversity, but housed in a general, common structure (Riveros-Rosas et al., 2019).

\section{GENERAL STRUCTURE}

\section{Introduction to the Aldehyde Dehydrogenase Common Architecture}

ALDH enzymes typically exist and function as homotetramers or homodimers (Figures 1A,B) formed by subunits of approximately $450-500$ amino acids to construct a $50-60 \mathrm{kDa}$ protomer. The overall structure consists of three distinct, conserved domains, the $\mathrm{NAD}(\mathrm{P})$ binding domain, the catalytic domain and the "arm-like" oligomerization domain Liu et al. (1997) establishing a domain fingerprint for this superfamily (Figure 1C). At the interface of these domains, buried within the enzyme, is a funnel-shaped cavity with an opening leading to the catalytic pocket. Which within harbors the important catalytic thiol, Cys residue.

\section{The Active Site}

The active site of the ALDH is located at the base of a hydrophobic, funnel shaped tunnel, close to the subunit's interface and opposite the cofactor binding site. A highly reactive active-site cysteine residue (Cys302 in ALDH1a1 and ALDH2, Cys243 in ALDH3a1, human numbering), which is highly conserved throughout the ALDH family, has been accepted as the catalytic thiol (Farres et al., 1995). The side chain of the Cys residue protrudes into the catalytic tunnel that extends through each subunit. The upper portion of the funnel between the catalytic Cys and the entrance, is made up of residues from all three domains. Furthermore, this passage is identified as a catalytic pocket, relating to where the aldehyde first forms a tetrahedral intermediate with a Cys residue to initiate catalysis. In addition to aldehyde oxidation, some ALDHs possess NAD esterase activity (Feldman and Weiner, 1972; Sládek, 2003; Vasiliou and Nebert, 2005). Interestingly these enzymes utilise the same active site residues to carry out this function as dehydrogenase catalysis, however esterase activity does not require the addition of the $\mathrm{NAD}(\mathrm{P})$ cofactor (Koppaka et al., 2012). The so-called "pseudoenzyme" human ALDH16 lacks this catalytic Cys residue, resulting in absence of catalytic activity, proposed to function as a binding protein (Liu and Tanner, 2019).

Conserved residues are present across the sequences of the ALDH members, highlighting vital, positional requirements for systematic catalysis by the enzyme. Sequence alignment of 145 known ALDHs demonstrated 4 invariant residues and 12 highly conserved residues (present in $95 \%$ of sequences analyzed). Of these, glycines and prolines were abundant ( 7 and 2, respectively), likely highlighting critical chain-bending points due to the antihelical and beta-sheet potentials of these residues (Perozich et al., 1999b). The catalytic Cys is conserved in all structures which harbor catalytic activity, the above mentioned ALDH16 being a prime example of a non-catalytic member (Liu and Tanner, 2019). In addition, two other important conserved residues associated with catalysis, lysine (Lys192) and glutamic acid (Glu268) (human numbering), are evident across the ALDHs. Glu268 is directly involved in catalysis providing a water molecule required for deprotonation of the catalytic thiol and 

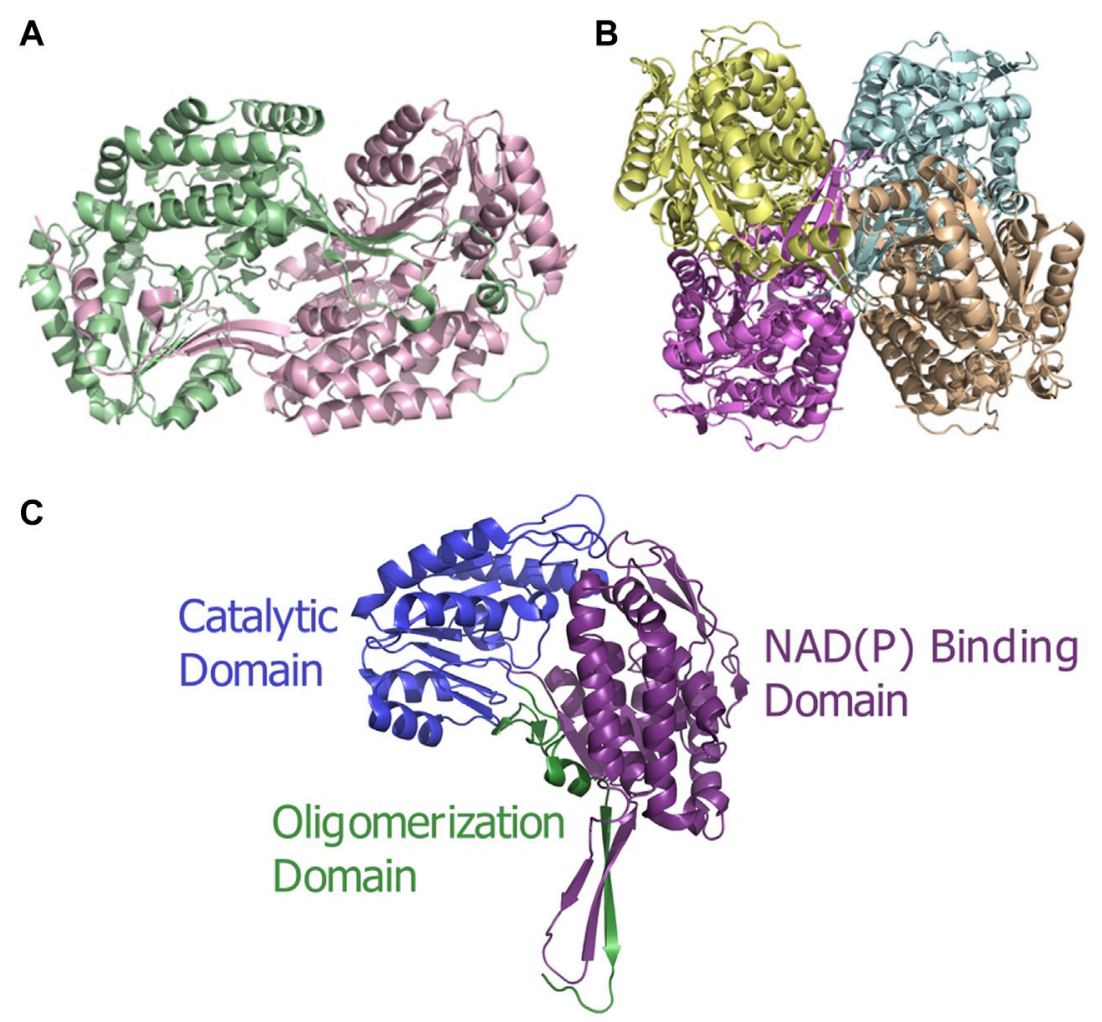

FIGURE 1 | ALDH architecture (A) Homodimeric structure of the human ALDH3A1 (PDB: 3SZA). (B) Homotetrameric structure of the human mitochondrial ALDH (PDB: 1NZX). (C) Three conserved domains illustrated on an ALDH monomer. The functional domains are highlighted: catalytic domain (blue), NAD(P) binding domain (purple), and oligomerization domain (green).

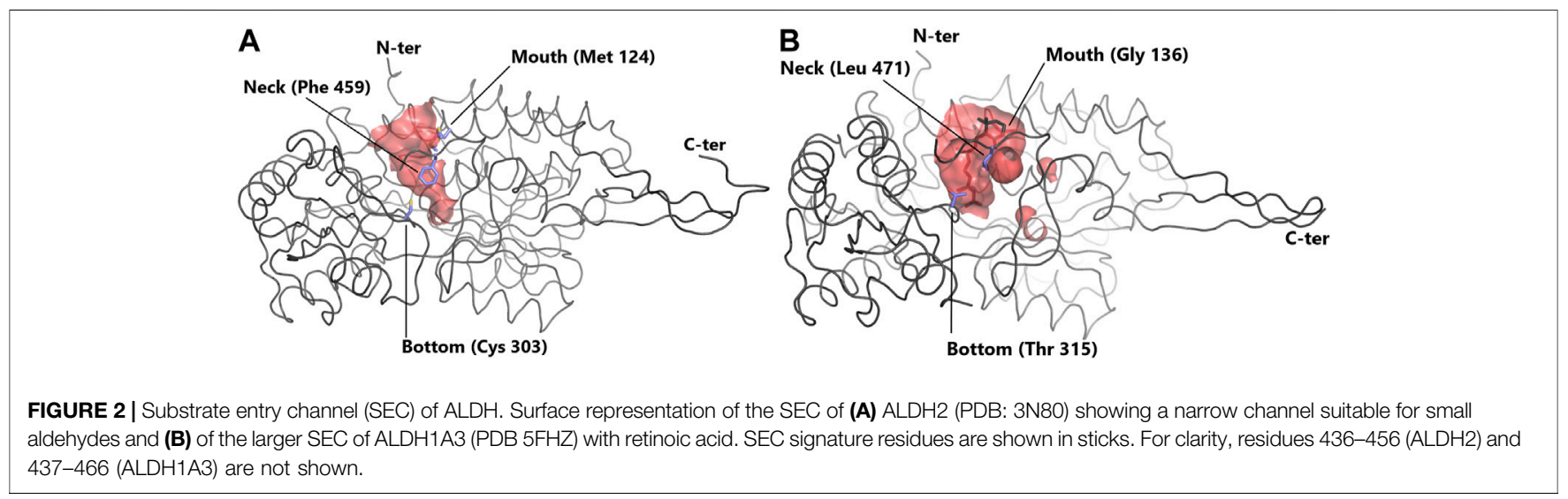

subsequent hydrolysis of the thioester intermediate (see details at the end of the section). Glu399 is also highly conserved and is involved in NAD binding along with Lys192, however, Glu399 is not as critical as Lys192, because during catalysis it is bound to the nicotinamide ring which appears to have to move during the catalytic process (discussed further below). An amino acid exchange of Lys192 causes an alteration in NAD binding and the rate-limiting step but substitution of Glu399 only alters the latter (Sheikh et al., 1997).
Contrasts in ALDH enzymes exist within the cavity used for entry of the substrate into the active site, called the substrate entry channel (SEC) (Sobreira et al., 2011). The size and shape of the SEC dictates the ability of the ALDH to process small or large aldehydes (Figure 2). Typically, ALDH1 has a larger SEC volume than ALDH2 (589 \pm 59 and $403 \pm 53 \AA^{3}$, respectively), consistent with their favored substrate. Human ALDH2 displays a narrow channel with a constricted entrance Steinmetz et al. (1997), one of its main substrates being acetaldehyde. In contrast sheep 
ALDH1A1 exhibits a large SEC equipped with a broad opening enabling access for larger aldehydes (Moore et al., 1998). This highlights that SEC topology influences substrate preferences within the ALDH family. ALDH2, with acetaldehyde as its natural substrate, displays no activity for retinaldehyde, while ALDH1 can oxidise retinaldehyde but can only inefficiently process acetaldehyde (Klyosov, 1996; Moore et al., 1998). Three key signature residues present in the SEC are responsible for geometry/function and thus substrate specificity. These residues are designated the "mouth" (124), "neck" (459) and "bottom" (303) (human ALDH2 numbering) (Figure 2) (Moore et al., 1998; Sobreira et al., 2011). The "mouth" residue performs a size selection function, bovine ALDH2 possesses a bulky Met and sheep ALDH1A1 and human ALDH1A3 a Gly (Moore et al., 1998)(Steinmetz et al., 1997; Moretti et al., 2016), resulting in either an open or narrow entrance to the SEC. The "neck" residue is present at the proximal third of the channel. In vertebrate ALDH2 this residue is a large Phe in contrast to ALDH1 where it is typically a smaller Val or Leu (Sobreira et al., 2011)(Moretti et al., 2016). The third signature, the "bottom", is located directly beside the catalytic Cys at the end of the channel. In vertebrate ALDH2s this residue is usually a Cys, whereas in ALDH1 it can be Thr, Ile or Val (Sobreira et al., 2011; Moretti et al., 2016). Within ALDH2 the "neck" and "bottom" residues (Phe459 and Cys303) assist in holding the smaller aldehyde substrate close to the catalytic Cys-a mechanism not required for the larger substrates in ALDH1. Favourable surface interactions between Cys303 ("bottom") and Phe459 ("neck"), fixates the position of the "neck" residue and in turn the position of Phe 465 which is responsible for holding the substrate close to the catalytic thiol. This mechanism is not present in ALDH1 due to the residues substitutions as highlighted above, further emphasising the specialisation of the SEC for preferred substrates (Sobreira et al., 2011). This highlights that the size of the key amino acid signatures dictate substrate specificity while also playing a key role in surface interactions for further SEC specialization. Interestingly it was demonstrated that metazoan class 1 and class 2 ALDHs are members of a single clade, with ALDH2 forming a subgroup nested within this ALDH1/2 clade. Throughout evolution, class 1 and 2 often switched between small and large SECs following gene duplication, transforming restricted small channels into wider ones and vice versa. Expansion of the channel occurred due to substitution of a bulky methionine residue with a small alanine or glycine, reducing steric hindrance effects (Sobreira et al., 2011).

The electrostatic potential of the SEC plays a significant role in dictating the range of substrates which can be utilised for oxidation. The binding site and channel are tailored, optimising the processing of certain aldehyde substrates. ALDHs that bind positive substrates, commonly display a negative electrostatic potential in the SEC and vice versa. In contrast, ALDHs that have non-polar substrates contain neutral electrostatic potential surfaces for easy, efficient conversion (Riveros-Rosas et al., 2013). For example, human ALDH1, with a physiological substrate, retinaldehyde, has a neutral SEC (Riveros-Rosas et al., 2013; Moretti et al., 2016). ALDHs who use positive betaine aldehydes as their substrates e.g., ALDH9 from Pseudomonas eruginosa (PDB: 2WME) and ALDH25 from Staphylococcus aureus (PDB: 4MPB) are equipped with negatively charged SECs (González-Segura et al., 2009; Riveros-Rosas et al., 2013; C. Chen et al., 2014). In addition, ALDH11 from Streptococcus mutans (PDB: 1EUH) uses glyceraldehyde-3-phosphate as its substrate and displays a positive electrostatic potential at the SEC (D'Ambrosio et al., 2006). In contrast human ALDH3 (PDB: 3SZB), demonstrating a negatively charged SEC is known to convert medium to long chain aliphatic and aromatic aldehydes raising the question of the possibility of an unidentified positively charged substrate for this enzyme (Muzio et al., 2012; Riveros-Rosas et al., 2013).

Systematic catalysis performed by an ALDH occurs in five distinct steps within the active site, 1) activation of the catalytic thiol, Cys302 or equivalent, using a water molecule for watermediated deprotonation by Glu268, 2) consequential nucleophilic attack on the electrophilic aldehyde by the thiol group of the catalytic cysteine, 3) formation of a tetrahedral thiohemiacetal intermediate, via deacylation, coupled with concomitant hydride transfer to the pyridine ring of $\mathrm{NAD}(\mathrm{P})$, 4) hydrolysis of the resulting thioester, 5) dissociation of the reduced cofactor producing $\mathrm{NAD}(\mathrm{P}) \mathrm{H}$ and regeneration of the enzyme by $\mathrm{NAD}(\mathrm{P})$ binding (Figure 3 ). It is proposed that an ordered water molecule plays an essential role in facilitating catalysis. This water molecule is required to be bound to the side chain of Glu268 to allow for both the deprotonation of the catalytic thiol and subsequent hydrolysis of the thioester intermediate (steps one and four highlighted above) (Koppaka et al., 2012).

\section{NAD(P) Cofactor Choice and Utilisation}

A selection of enzymes use dinucleotide cofactors, such as NAD or flavin adenine dinucleotide (FAD), and even though their overall enzyme structures may differ they are normally equipped with dinucleotide binding domains consisting of either a Rossmann fold (Rossmann et al., 1974; Wierenga et al., 1986) or a $(\alpha / \beta)_{8}$ barrel structure (Wilson et al., 1992; Hoog et al., 1994). The cofactor-binding domain in ALDH is composed of a Rossmann fold. The pyrophosphate moiety of the cofactor makes close contact with the first $\beta-\alpha-\beta-\alpha-\beta$ of the Rossmann fold, specifically the loop between $\beta 1$ and $\alpha \mathrm{A}$ (Figure 4A). The helix $\alpha \mathrm{A}$ has been termed the "dinucleotide binding helix" (Hol et al., 1978) due to the helix dipole providing a favourable interaction with the negatively charged pyrophosphate moiety of the dinucleotide molecule (Liu et al., 1997).

In NAD binding, binding of the adenine part of the cofactor is conserved across ALDHs in a fixed conformation, with no movement necessary during catalysis. In contrast, the nicotinamide portion appears to be flexible, contributing to variable conformations throughout superfamily members (Figure 4B). Movement of the nicotinamide in and out of the active site during catalysis is a conserved and important mechanism of functional ALDHs. The adenine ring of the $\mathrm{NAD}(\mathrm{P})$ molecule during binding resides in a hydrophobic pocket between the helices, $\alpha F$ and $\alpha G$, as observed in structures of sheep ALDH1, bovine ALDH2 and rat ALDH3 


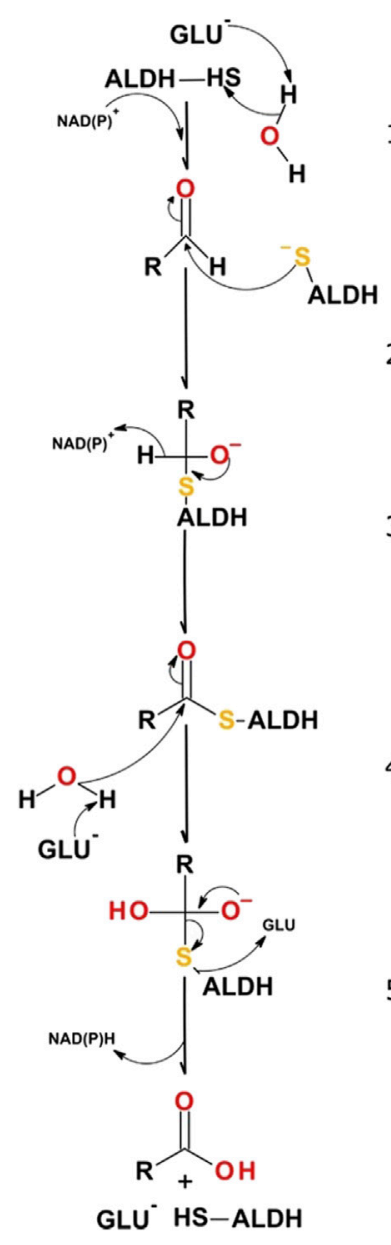

1. Activation of the catalytic Cys by an
ordered $\mathrm{H}_{2} \mathrm{O}$ and Glu via proton abstraction.

2. Nucleophilic attack by the thiolate group of the Cys on the carbonyl of the aldehyde.

3. Creation of tetrahedral thiohemiacetal intermediate via deacetylation. Hydride transfer from the intermediate to the pyridine ring of $\mathrm{NAD}(\mathrm{P})^{+}$.

4. Hydrolysis of the thioester intermediate. $\mathrm{H}_{2} \mathrm{O}$ makes nucleophilic attack on the carbonyl removing the Sulfur as a leaving group.

5. Dissociation of the reduced cofactor. Regeneration of the enzyme via the addition of $\mathrm{NAD}(\mathrm{P})^{+}$.

FIGURE 3 | ALDH reaction mechanism highlighting the five essential steps in the catalytic scheme.

(Liu et al., 1997; Steinmetz et al., 1997; Moore et al., 1998). Stabilisation of the adenine ring within the enzyme is conferred by cradling of the ring using residue sidechains and hydrogen bonds. In both ALDH1 and ALDH2 structures the adenine ribose forms hydrogen bonds with Lys192, Glu195 and Ile166. The specific amino acid sequence, $\mathrm{G}_{1} \mathrm{XG}_{2} \mathrm{XXG}_{3}$, which reflects the turn at the end of the first $\beta$-strand, interacts with the adenine ribose of the NAD molecule (Wierenga and Hol, 1983; Hempel et al., 1993). The nicotinamide portion of the cofactor seemingly demonstrates discrete disorder during binding leading to a number of cofactor conformations. In contrast to bovine $\mathrm{ALDH} 2$ and rat ALDH3 the nicotinamide ring binding in sheep ALDH1 was different (Moore et al., 1998). Two major conformations of the nicotinamide half were observed however with the less occupied conformation mimicking bovine ALDH2 structure. The specific differences between the ALDH1 and ALDH2 are realised, the nicotinamide ring of ALDH1 occupies nearly the same position as the nicotinamide ribose in ALDH2, a shift of over $5 \AA$ (Figure 4B). In addition, the same amino acids, Gln349, Glu399 and Phe401, confer the nicotinamide portion stabilisation in both ALDH1 and ALDH2 however they do so in a very different manner.
Flexibility of the nicotinamide is also assisted by the intentionally weak binding of the pyrophosphate moiety in ALDHs. In contrast to other NAD-dependent dehydrogenases there are few interactions between the negative phosphates and the protein residues, especially due to lacking lysines or arginines in the pocket. Instead, the interactions occur with a patch of strong negative electrostatic potential near the phosphate binding pocket conserved in ALDH1 and ALDH2. These interactions act like a ball and socket joint conferring flexibility within the molecule. The rational for nicotinamide flexibility requirements relate to the water mediated deprotonation using Glu268 during catalysis, with the Glu268 also exhibiting disorder. Specifically, during the hydride transfer step the side chain of Glu268 must be tucked away from the nicotinamide ring of the cofactor. Before deacylation of the thioester can occur, the nicotinamide portion must at least half exit the active site to allow for room for the water molecule to position itself near the thioester carbon of the acyl intermediate. Flexibility and variable conformations of both Glu268 and the nicotinamide ring are paramount for proper dehydrogenase action by ALDH.

The specificity of the enzyme to utilise a sole cofactor is dictated by distinct features within the cofactor-binding 

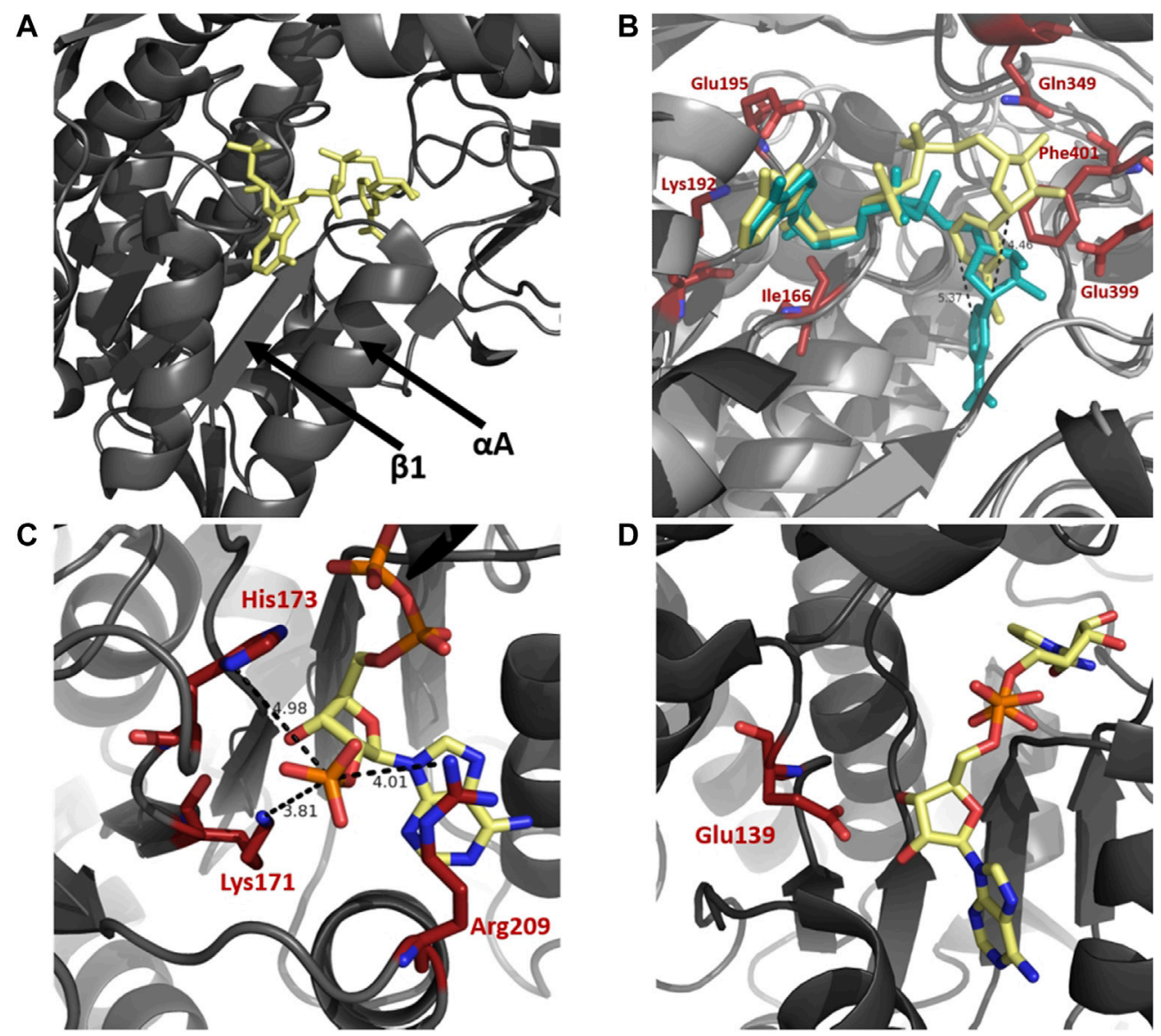

FIGURE 4 | Cofactor binding mechanism of ALDH. (A) Depiction of $\alpha A$ and $\beta 1$ of the Rossmann fold in the cofactor binding domain. (B) Varying conformations of the nicotinamide portion and constant orientation of adenine ring of NAD demonstrated on sheep ALDH1 and bovine ALDH2 (PDB: 1BXS and 1A4Z). ALDH1 (dark gray) and ALDH2 (light gray) are modeled as an overlay with NAD in yellow and blue, respectively. Measurements demonstrate an approximate $5 \AA$ shift of the nicotinamide portion. Key cofactor binding residues are highlighted in red. Residues 241-253 have been omitted for visualisation purposes. (C) NADP 2'phophate shown in close proximity to Lys171, His173, and Arg209, no acidic residue is present in the NADP dependent ALDH from V. Harvei (PDB: 1EYY). (D) Human ALDH3A1 (PDB: 4L2O), a non-obligatory NADP ALDH, shows a NAD cofactor in close proximity to Glu139 even though this ALDH can utilise NADP.

domain. The Rossmann fold commonly contains an acidic residue located at the end of $\beta 2$, which seemingly dictates cofactor preference. ALDH enzymes that favor the use of NADP may possess positively charged amino acids that stabilise the negative $2^{\prime}$ phosphate moiety. Absence of an acidic residue may prove essential for preference of NADP, as if present, repulsion effects would occur between the two negative charges (Perozich et al., 2000). The presence of an acidic residue in close proximity to the position of $2^{\prime}$ phosphate may dictate the cofactor specificity as it does not allow NADP to be stable within the active site, deeming the enzyme preferable to NAD (Sharkey et al., 2013). From structural analysis, human ALDH2 and ALDH from Thermus thermophilus $\left(\mathrm{TtALDH}_{530}\right)$ both possess a glutamic acid residue in close proximity to the 2' phosphate when using NADP, while both enzymes prefer NAD. ALDH from Vibrio Harveyi and Pyrobaculum sp. both lack a Glu residue, with $V$. Harveyi possessing an adjacent lysine residue, both utilise NADP preferably (Figure 4C). In contrast human ALDH3A1 and ALDH3A2 can use NADP but are not obligatory NADP enzymes. They are shown to possess the corresponding acidic, glutamate residue at the correct position despite their use of NADP as a cofactor (Sharkey et al., 2013) (Figure 4D).

\section{Structural Analysis of Oligomerization State: Dimer vs. Tetramer vs. Hexamer}

As previously stated ALDHs exist in dimeric or tetrameric state, but a small number of resolved structures have now demonstrated the presence of hexameric enzymes. ALDHs are constructed by domain swapped dimerization with tetramers typically being formed by a dimer-of-dimers (Figure 1). A resounding question within the literature asks, what dictates the oligomeric state which an ALDH enzyme adopts? In dimeric ALDHs, an extension of approximately 17 amino acids at the C-terminus in the form of a tail occurs. When compared to their tetrameric counterparts a 56 amino acid deletion at the $\mathrm{N}$-terminus is present (Figures $\mathbf{5 A}, \mathbf{B}, \mathbf{6 A}$ ). It has been tacitly assumed that the presence or absence of a C-terminal tail within 


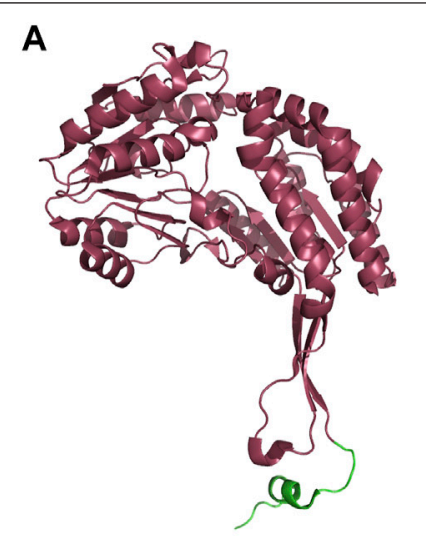

D

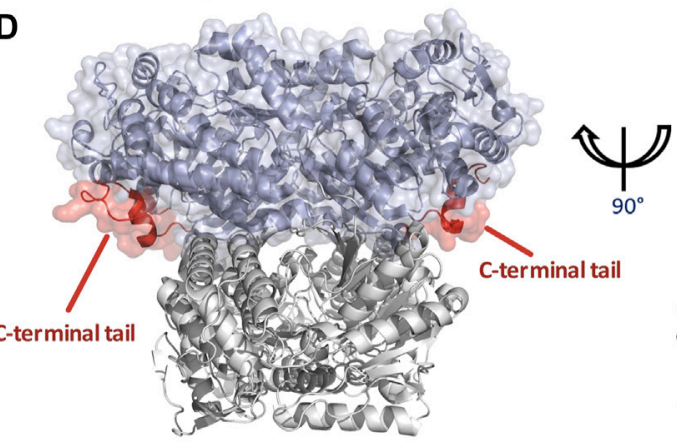

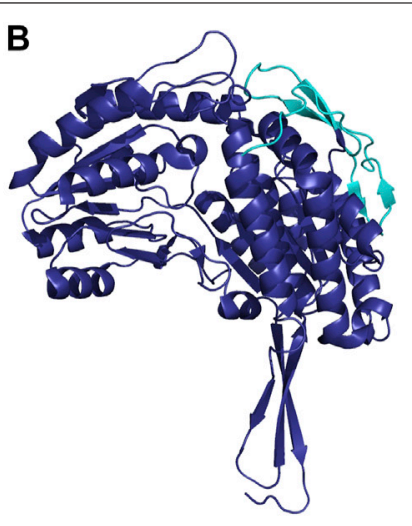
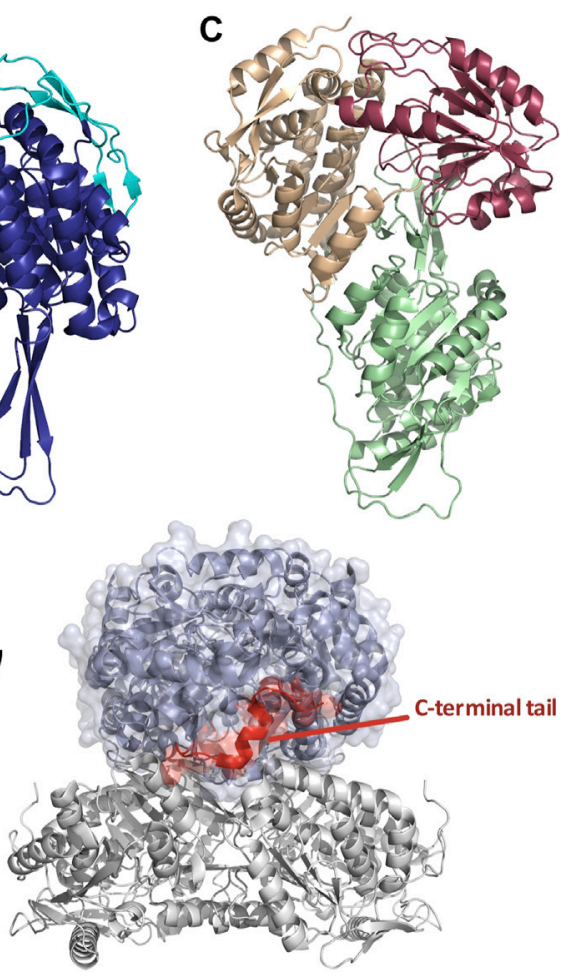

FIGURE 5 | Structural features of dimeric and tetrameric ALDHs. (A) The C-terminal tail extension of dimeric ALDHs is represented in green against the monomer of ALDH3A1 (Red, PDB: 3SZA). Note the deletion of the first 56 amino acids in comparison to image (B) of the tetrameric ALDH2 (cyan). (B) The "so-called" $\mathrm{N}$-terminal extension is represented in cyan against the monomer of ALDH2 (Blue, PDB: 1NZX). Note the absence of the C-terminal extended tail. (C) LsALDH16 monomer highlighting the NAD binding domain (orange), the catalytic domain (red) and an extra structural domain (green). (D) Surface representation of the dimeric ALDH3A1 (light blue, PDB: 3SZA) superimposed on ALDH2 (Gray, PDB: 1NZX). The C-terminal tail of ALDH3A1 is depicted in red. For clarity, only the opposing dimer of ALDH2 is shown.

FIGURE 6 | Graphical representation of the architecture of ALDHs. (A) ALDH class 1 and 2 being defined by a set of $\mathrm{N}$-terminal residues (yellow) and the lack of the C-terminal tail (green). ALDH class 3 being defined by the absence of $\mathrm{N}$-terminal residues and presence of the $\mathrm{C}$-terminal extension. Note that the TtALDH ${ }_{530}$ contains both this $\mathrm{N}$-terminal segment and the extended C-terminus with LSALDH16 containing a C-terminus constructed by a non-functional Rossmann fold domain. Modified from Hayes, et al. (Hayes et al., 2018). (B) Representation of ALDH functional domains on a typical ALDH and AdhE spirosome of 3 subunits. Note: size of the graphic representation does not directly relate to the size of the domains.

the enzyme dictates the oligomeric mode, between a dimer or a tetramer. Indeed, in the dimeric structures the C-terminal tail extends into the region where a second dimer pair would assemble and, thus, disrupting tetramer formation (Hurley et al., 1999; Rodriguez-Zavala and Weiner, 2001). Figure 5D demonstrates the position of the dimeric ALDH3 C-terminal tail 

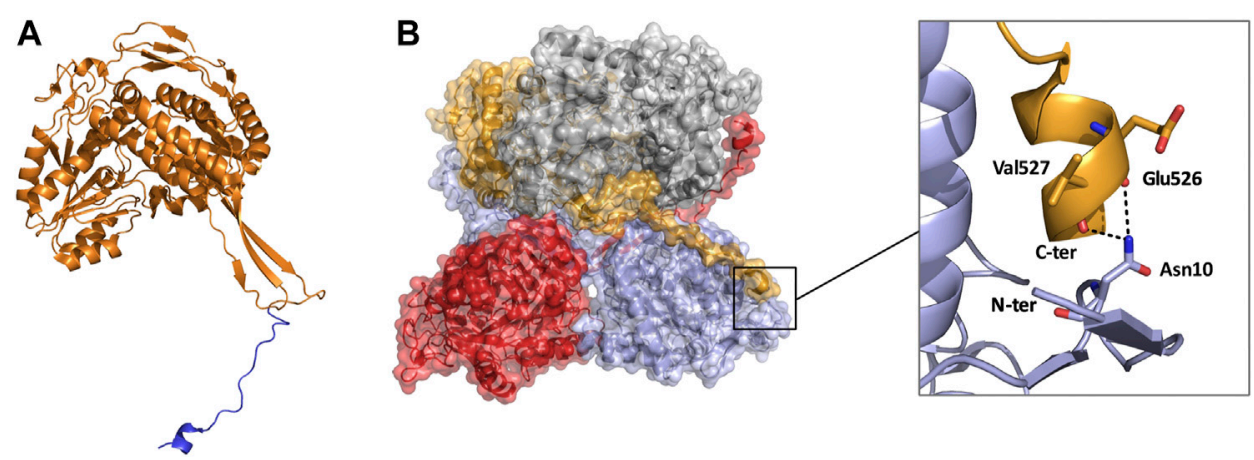

FIGURE 7| Monomeric and tetrameric structure of TtALDH 530 (PDB: 6FJX). (A) Monomer highlighting the C-terminal extension in blue. (B) Surface representation of the tetrameric assembly, note how the $\mathrm{C}$-terminal tail from one monomer wraps its diagonal monomer and interacts with its $\mathrm{N}$-terminus. Monomers are represented in contrasting colors.

in comparison to a tetrameric ALDH2 organisation. Here it is demonstrated that the orientation of the tail is modeled toward a region to the outer sides of the dimer. Studies which eliminated the C-terminal tail from the dimeric structure demonstrated that a tetramer was formed at low salt conditions but reverted back to a dimer upon increase of ionic strength (Rodriguez-Zavala and Weiner, 2001). Also, the addition of 5 and 17 amino acids at the C-terminus of a tetrameric isozyme subunit was trialled, but conversion to a dimer was not achievable. When 5 residues were added, tetrameric structure was conserved but activity was reduced to $30 \%$ compared to wild type. When 17 residues were added this greatly decreased the stability of the enzyme (Rodriguez-Zavala and Weiner, 2001). This demonstrates that the C-terminal tail contributes to quaternary arrangement in dimeric structures but does not affect tetrameric ones. However, the tail is not the sole factor holding the dimer together, as upon removal and high ionic strength the dimer was conserved, suggesting other favourable interactions such as between single residues or inter-domain interactions.

Until recently all tetrameric ALDHs were organized as dimer of dimers free of C-terminal extensions. However, emerging research suggests the contrary. The novel ALDH from T. thermophilus HB27 $\left(\mathrm{TtALDH}_{530}\right)$ Hayes et al. (2018) showed an interesting feature with an unusual extended C-terminal tail compared to available structures (Figure 7). In contrast to other ALDHs, this extended tail contributes to the tetrameric assembly and the stability of the protein as it completely wraps the opposing monomer. This results in the formation of a network of salt bridges and hydrogen bonds with the $\mathrm{N}$-terminal residues and oligomerization domain of the opposite monomer. As the tail wraps the opposing monomer, it is dragged across the opening of the substrate entry tunnel conferring possible roles in active site regulation. In addition, an ALDH from Pseudomonas putida (PpALDH) contains an extension in its oligomerization domain associated with "hugging" its neighboring monomer and interacting with its active site (Crabo et al., 2017). This enzyme contains an additional 6 residues in comparison to its closest related homolog, the sheep liver ALDH1, and is associated with occluding active site entrance perhaps conferring substrate specificity. This enzyme ultimately preferred smaller aldehyde substrates. Similarly, the recently resolved structure of an ALDH16 from Loktanella sp. (LsALDH16) demonstrated a unique oligomerization mode and potential regulation of catalysis utilising a C-terminal extension (Liu and Tanner, 2019). ALDH16 shows what has been described as transhierarchical structural similarity, where tertiary interactions within one protein mimic quarternary interactions in another. Indeed, ALDH16 features the classical NAD and catalytic domains in addition to a large non-functional cofactor binding domain in the C-terminal (Figures 5C, 6A). In this case the overall structure of LsALDH16 mimics the classical tetrameric organisation, although being formed by only two subunits.

The first description of a membrane associated ALDH, the dimeric fatty aldehyde dehydrogenase (FALDH), displayed an unrecognised C-terminal, hydrophobic "gatekeeper" helix with a function in active site restriction and substrate specificity (Keller et al., 2014). The helix induces a $93^{\circ}$ kink toward the SEC. Adjacent is a transmembrane helix which typically anchors the FALDH to the endoplasmic reticulum or peroxisomes assisting in processing of long chain aldehydes generated in the membrane which are not accessible to cytosolic enzymes. Once the FALDH is embedded in the membrane the active site funnel and "gatekeepers" are oriented toward the membrane, with the cofactor domain toward the cytosol. "Gatekeeper" residues form a hydrophobic ring around the entrance to the SEC, possibly allowing for entry or fusion with membranes to facilitate efficient substrate binding. Removal of the gatekeeper helix resulted in reduced capacity for hexadecanal but not toward shorter aldehydes such as octanal (Keller et al., 2014). Recently a similar C-terminal "gatekeeper" was observed in the Staphlococcus aureus ALDH (SaALDH) but with the characteristic kink orientating the helix away from the SEC (Tao et al., 2020). Upon binding of substrate a conformational change associated with the C-helix is adopted, a change in direction of the kink by $10.6^{\circ}$ now orients the "gatekeeper" toward the SEC. Without substrate, in SaALDH, the C-helix takes an open conformation allowing for substrates 

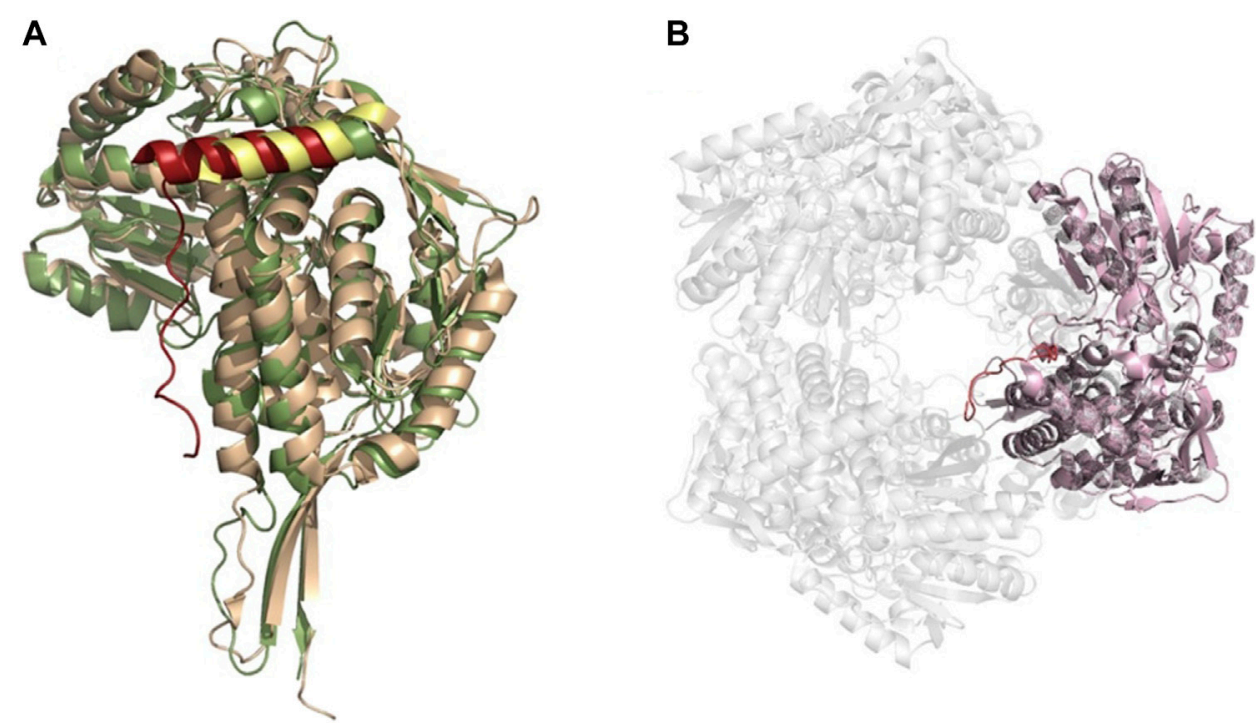

FIGURE 8|ALDH hexameric structure (A) Superimposition of the monomers of yeast ALDH4A1 (orange) and TtP5CDH (green) (PDB: 4OE6, 2BHQ, respectively), demonstrating the N-terminal extension equipped with an alpha helix in hexamer forming ALDHs. Extension is shown in red for TtP5CDH and yellow for yeast. (B) The hexameric assembly of TtP5CDH (PDB: 2BHQ) with the N-terminal loop (red) penetrating the pore formed by the hexamer.

to enter, whereas with bound substrate the C-helix locks the aldehyde in place and adopts a closed conformation.

A third oligomeric state of ALDHs exists in the form of a hexamer (Figure 8), organized as trimer of dimers. However, this is a less common state and there are only two resolved structures from S. cerevisiae (ScALDH4A1, 4OE6) and T. thermophilus (TtP5CDH, pyrroline-5-carboxylate dehydrogenase, 2BHQ), with another unavailable structure from Deinococcus radiodurans being reported (Inagaki et al., 2006; Luo et al., 2013; Pemberton et al., 2014). No mammalian hexameric structures have been reported to date. Work on TtP5CDH showed that the formation of the hexamer is mainly associated with the presence of a hexamerization hotspot. An arginine residue was identified in the bacterial model while a tryptophan residue assumes this role in ScALDH4A1 (Pemberton et al., 2014). Without this essential hotspot residue, hexamer formation is compromised (Luo et al., 2013). Interestingly, through our own structural analysis, a unique feature of the hexamer structure was identified. In $\mathrm{TtP} 5 \mathrm{CDH}$, a 33 amino acid extension of the N-terminus is evident, consisting of a loop of 15 amino acids penetrating the pore formed by the hexameric assembly and interacting with the adjacent subunit, while the remaining 18 residues are organized in an alpha helix. In comparison, the yeast ScALDH4A1 shows a similar alpha helix, however, with the first 30 amino acids not visible in the structure due to disorder (Figure 8).

\section{Alcohol Dehydrogenase-Aldehyde Dehydrogenase Bifunctional Spirosomes}

Aldehyde-alcohol dehydrogenase (AdhE) is a bifunctional enzyme, key to bacterial metabolic processes in physiology and pathology, which contains two enzyme domains, ALDH and alcohol dehydrogenase $(\mathrm{ADH})$. While monofunctional ALDH and $\mathrm{ADH}$ are found in all kingdoms of life, AdhE is mostly found in bacteria and some unicellular eukaryotes such as microalgae. These helical macromolecule assemblies, called spirosomes, were first discovered in 1975 Kawata et al. (1975) and were more recently designated as filaments (Pony et al., 2020). A recent, in depth study of the E. coli AdhE was carried out by Kim et al. highlighting their critical structure-function relationship (Kim et al., 2019). The AdhE monomer $(96.1 \mathrm{kDa})$ is composed of an $\mathrm{ALDH}$ and $\mathrm{ADH}$ domain separated by a linker of 7 amino acid residues (Figure 6B). Together with the protruding $\beta$-turn from the ALDH domain the linker forms a 3 stranded $\beta$-sheet for connection of the two catalytic domains. The structures of the $\mathrm{ALDH}$ and ADH domains are similar to other known structures, both contain two lobes, the ALDH with a Rossmann fold and NADH binding cleft, while the ADH contains an $\mathrm{Fe}^{2+}$ and NADH binding pocket. Two AdhE monomers form a dimer in a head-to-head arm crossing fashion. To then form the spirosome structure, dimers are almost stacked one on top of each other, through use of hydrophobic interactions mediated by the ADH domains. For example, six AdhE molecules with two $\mathrm{ADH}$ domains at either end will consist of approximately 1.5 helical turns. Repetition of the helical unit leads to spirosome formation (Figure 9).

It is determined that the spirosome structure is crucial to maintain catalytic activity of the AdhE, with the ALDH and ADH activity topologically separated due to the helical filamentous structure (Kim et al., 2019). Within the structure, the ALDH and $\mathrm{ADH}$ domains from different subunits are clustered together rather than intertwined with mismatching domains. This allows for dividing of activity and the ability for the ALDH and ADH to work solely and more efficiently. The ALDH active site is located toward the outer surface of the helical structure, while the ADH 


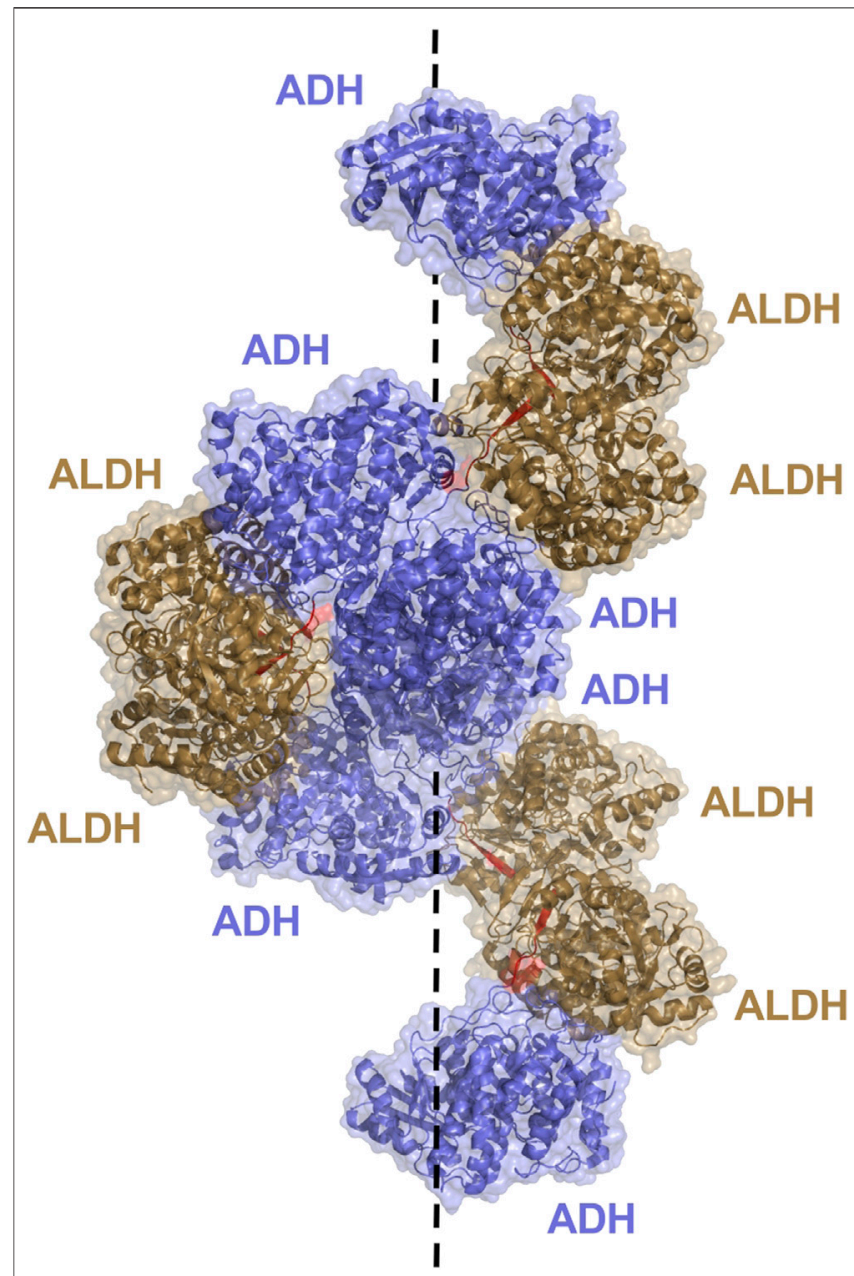

FIGURE 9 | AdhE extended spirosome structure shown as a cartoon with transparent surface representation (PBD: 7BVP). ALDH domains located on the outer surface are shown in brown, $\mathrm{ADH}$ domains in blue on the inside of the spirosome and the 7 amino acid linker between domains in red. The dotted line demonstrates the helical axis of the spirosome.

active site resides toward the inner surface. Effective elimination of cytotoxic acetaldehyde is imagined. The acetaldehyde produced by ALDH will not enter the cell, it will be passed to the $\mathrm{ADH}$ within the spirosome structure for timely conversion to ethanol. Additionally, maintenance of the spirosome structure proved critical in AdhE catalytic activity. Disruption of spirosome formation and generation of free dimers through mutation led to a dramatic decrease in AdhE activity. It is notable that spirosome formation proved critical for maintenance of the forward ALDH reaction, conversion of Acetyl Co A to acetaldehyde, but no other reactions, suggesting that the spirosome might play a role in regulating the direction of AdhE activity.

The outer residing ALDH of the AdhE is exposed to solvent, whereas the $\mathrm{ADH}$ on the inside of the compact spirosome is not readily accessible. AdhE spirosomes undergo a structural transition in the presence of cofactor to assist with catalysis (Kim et al., 2020). A compact form is changed into an elongated, extended spirosome upon addition of cofactor
(Kessler et al., 1991; Kim et al., 2019). Further suggestions lead to the cofactor binding in the $\mathrm{ADH}$ domain being the most critical to initiate the change. This transition may be involved in regulation of AdhE activity. Substantial structural changes upon binding of the cofactor leads to a widely opened spirosome with an accessible $\mathrm{ADH}$ catalytic pocket. Moreover, the transition creates a substrate channel between the ALDH and ADH active sites, from two different subunits, which is accessible to solvent and thus substrates and products (Kim et al., 2020). In this conformation the otherwise restricted $\mathrm{ADH}$ active site is accessible to solvent from both outside and inside, with the inter-domain channel likely playing a role for transition of intermediate products, assisting with the bifunctional cascade reaction of the spirosome.

\section{STRUCTURAL PERSPECTIVE OF ALDEHYDE DEHYDROGENASE RELATED DISEASES}

ALDH enzymes are diverse in their function across all kingdoms of life, contributing to detoxification Esterbauer et al. (1991), Kelson et al. (1997), Choudhary et al. (2005), Ho and Weiner (2005), Rodríguez-Zavala et al. (2006), Marchitti et al. (2007), Shin et al. (2009), Jackson et al. (2011), Ouyang et al. (2020), biosynthesis Yoshida et al. (1992), Mellema et al. (2002), Kim et al. (2015), Pequerul et al. (2020), and non-enzymatic functions such as anti-oxidant Estey et al. (2007), Lassen et al. (2008), Marchitti et al. (2011), Singh et al. (2013), Voulgaridou et al. (2020), structural Piatigorsky (1998), Voulgaridou et al. (2017) and regulatory mechanisms Moreb (2008), Vassalli (2019), Voulgaridou et al. (2020) (Table 1). Proper function of these enzymes is essential for maintenance of cell function and survival, with prominent ALDH-linked diseases arising from mutation, catalytic knockout and structural disruption. Knowledge of the molecular and structural basis for diseases is critical for understanding pathology and ultimately for therapeutic design, typically via comparison of wild type and mutants. However, with regards to $\mathrm{ALDH}$ disease in literature, focus is mainly upon phenotype and not enzyme structure. A detailed breakdown of human ALDH related disease is shown in Table 2 (Chao et al., 1994; Dupé et al., 2003; Deak et al., 2005; Hui et al., 2007; Jo et al., 2007; Lassen et al., 2007; Husemoen et al., 2008; Marchitti et al., 2008; Pavan et al., 2009; Guo et al., 2010; Palo et al., 2010; Palmfeldt et al., 2011; Sulem et al., 2011; Sass et al., 2012; Vasiliou et al., 2013). Herein, we summarise and discuss previously studied ALDH diseases in context of mutations and their effect on ALDH structure and function. Disease causing mutations present in the ALDH superfamily can be arranged into three groups, these will be designated for the purpose of this review as 1) those that affect NAD binding, 2) those that affect the substrate binding site and 3) those that disrupt quarternary structure formation.

ALDH2. The mitochondrial ALDH2, plays a significant role in ALDH-linked pathology and may be the most extensively discussed ALDH within literature in terms of disease. Specifically, the single nucleotide polymorphism E487K Farrés et al. (1994), Larson et al. (2007) residing in the oligmerization domain, 
TABLE 1 | ALDH function, categorically highlighting examples of contribution to specific mechanisms.

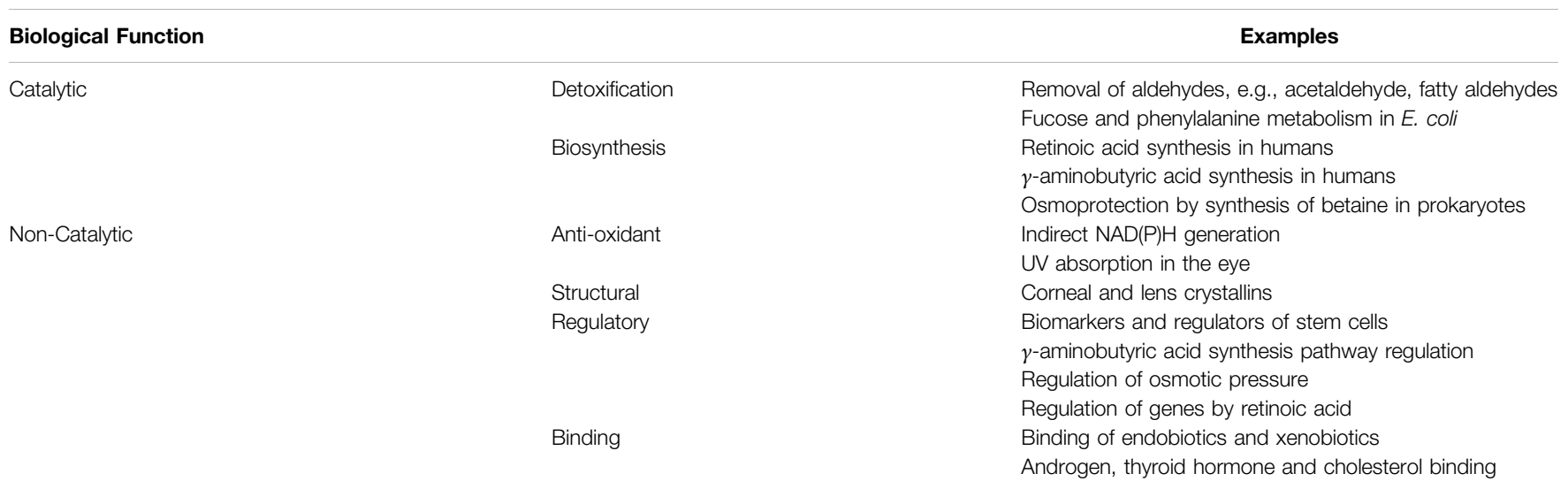

TABLE 2 | Human diseases linked to ALDH genes.

\begin{tabular}{ll}
\hline Gene & \\
\hline Aldh1a1 & Cataract formation; Parkinson's disease \\
Aldh1a2 & Disrupted embryonic development; spina bifida; rare cases of congenital heart disease \\
Aldh1a3 & Disrupted embryonic development \\
Aldh1b1 & Hypertension; ethanol sensitivity; ethylmalonic encephalopathy; bipolar disorder \\
Aldh2 & Alcohol intolerance; heart attack; hypertension; cancers; liver cirrhosis; Parkinson's and late onset Alzheimer's disease \\
Aldh3a1 & Cataract formation \\
Aldh3a2 & Sjogren-Larsson syndrome \\
Aldh4a1 & Type Il hyperprolinemia \\
Aldh5a1 & $\gamma$-hydroxybutyric aciduria \\
Aldh6a1 & Psychomotor delay; methylmalonic aciduria \\
Aldh7a1 & Pyridoxine-dependent epilepsy; osteoporosis \\
Aldh16a1 & Gout \\
Aldh18a1 & Hyperammonemia
\end{tabular}

resulting in the $\mathrm{ALDH} 22^{\star} 2$ variant, is related to complications in cardiovascular disease Chen et al. (2019), cancer Seitz and Stickel (2010), alcohol intolerance Chang et al. (2017) and late onset Alzheimer's disease (Kamino et al., 2000). ALDH2 2 can be designated as group 1 and 3 in relation to structure-function disruption. When just one protomer of the tetrameric assembly contains $\mathrm{ALDH} 2 * 2, \mathrm{NAD}$ binding is altered and catalytic activity is lost, resulting in accumulation of cytotoxic acetaldehyde (Larson et al., 2005). This mutation is responsible for a 200fold increase in $\mathrm{K}_{\mathrm{M}}$ for $\mathrm{NAD}$ and a 10 -fold reduction in kcat, ultimately severely reducing catalytic rate in-vivo. Physiological levels of NAD cannot reach the demand of the ALDH $2 * 2$. Within $\mathrm{ALDH} 2$, the adenosine portion of NAD is nestled within the cleft present between $\alpha \mathrm{F}$ and $\alpha \mathrm{G}$ of the Rossmann fold. The $\alpha \mathrm{G}$ helix is situated at the dimer interface and interacts with its same helix from the opposing subunit. Glu487 forms hydrogen bonds with Arg264 and Arg475 located on the C-terminus of $\alpha-\mathrm{G}$ and within a loop on the opposing subunit respectively. With the E487K substitution, stabilisation of $\alpha \mathrm{G}$ and the loop containing Arg475 is lost, leading to dimer destabilisation, NAD binding site disorder and some active site residue repositioning. Specifically, residues Glu399 and Glu268 essential in coenzyme positioning and catalysis respectively. A clinically relevant, specific activator of
ALDH $2 * 2$ named Alda-1, was found to enhance enzyme activity in-vitro and in-vivo. Importantly Alda-1 restores activity lost by the $\mathrm{E} 487 \mathrm{~K}$ mutation, through binding at the entrance of the catalytic tunnel, dramatically decreasing $\mathrm{K}_{\mathrm{M}}$ for cofactor and increasing $\mathrm{V}_{\max }$ (Chen et al., 2008; Perez-Miller et al., 2010). E487K substitution in ALDH2 causes a loss of electron density at helix $a \mathrm{G}$, and active site loop containing Arg475. As revealed by the crystal structure of Alda- 1 in complex with $\mathrm{ALDH} 2^{\star} 2$, binding of Alda-1 restores the aG structure and the loop even though Alda-1 has no direct contact with these residues (PerezMiller et al., 2010). Alda-1 is therefore an agonist and simultaneously functions as a chemical chaperone, exerting allosteric effect to restore the structural defect of a catalytically impaired enzyme (C.-H. Chen et al., 2014).

ALDH7A1. ALDH7A1 is responsible for lysine catabolism and its improper function is related to a seizure causing disorder named pyridoxine-related epilepsy (PDE), linked with approx. 60 missense mutations Stenson et al. (2008); van Karnebeek et al. (2016), Coughlin et al. (2018) which are highly considered within the literature. Interestingly, the classification of the mutations led to different symptom severity and treatment options in patients. Group 1 allowed for complete seizure control and normal developmental outcome, group 2 complete seizure control but 
developmental delay and group 3 showed persistent seizures with developmental delay. This suggests that multimer disruption is most detrimental in terms of ALDH7A1 dysfunction. Many of the associated mutations are surface accessible suggesting a role in retention of tetrameric assembly (Scharer et al., 2010). A study investigating 6 mutations (A129P, G137V, G138V, A149E, G255D, G236E) on ALDH7A1 present at the tetrameric interface and remote from the active Cys (18-28 $\mathrm{A}$ ) abolished enzyme activity indirectly and disrupted tetramer formation (Korasick et al., 2017). The most common missense mutation in ALDH7A1 has been reported as E427G Coughlin et al. (2018), occurring in $30 \%$ of PDE patients Mills et al. (2006), Plecko et al. (2007), Mills et al. (2010), a mutational hotspot for disease. A study investigating structural changes of ALDH7A1, exhibiting mutations E427G, E427Q and E427D, demonstrated a catalytic defect and a non-natural conformation of the NAD cofactor and as a result no catalytic activity (Laciak et al., 2020). The NAD adopts a flexible conformation and lacks a defined pose for E427G and $\mathrm{E} 427 \mathrm{Q}$ variants whereas an inactive pose is demonstrated for E427D, compromising catalysis due to lack of stabilisation and increased distance of the cofactor from active site Cys. In addition, this study again demonstrates the criticality of oligomerization for correct function with all three mutant variants compromised in tetramer assembly.

FALDH/ALDH3A2. ALDH3A2 is an endoplasmic reticulum bound FALDH responsible for the conversion of fatty aldehydes to fatty acids. Sjogren Larsson syndrome is a genetic disorder characterised by scaling skin, speech abnormalities, intellectual disability and spasticity caused by an autosomal recessive mutation in ALDH3A2, resulting in accumulation of aldehydes (Cho et al., 2018). Catalytic site mutations are mostly found in exon 4 with most exons harboring protein misfolding mutations. Interestingly the most frequent mutations, totalling 16, occur in exon 9, relating to coding for the C-terminal "gatekeeper" helix. As previously mentioned this "gatekeeper" assists in selection and easy processing of medium to long fatty aldehydes. Realised pathology could be associated with alterations in this helix as it may cause a shift in substrate specificity, inhibiting the removal of fatty aldehydes from the cell.

\section{CONCLUDING REMARKS}

The ongoing ALDH structure-function investigation is important for elucidating the novel features of these enzymes as well as the underlying mechanism for the cause of many diseases. Advancement in recent years lead to the understanding of new oligomerization modes, domains, extensions and bifunctionality contributing to both structure and function. The evolutionary progress of these enzymes clearly shows their adaptation for tailoring of the enzyme structure for processing of defined substrates: spirosomes for efficient shuttling of substrates between two enzyme domains within one structure conferring reduced cytoxicity, hydrophobic helices for selection of fatty aldehydes as well as membrane anchorage and appropriate geometry of the SEC for processing of the correct sized aldehyde. Vast fundamental knowledge of the ALDH has been paramount for the understanding of pathological diseases caused by ALDH. Deep understanding of human ALDH catalytic mechanisms, cofactor binding and geometry of the active site and SEC has allowed for in some cases complete characterisation of disease models in terms of diagnosis, development, biochemistry and even ALDH structural mechanisms. As an ever growing superfamily of enzymes, new characteristic features will develop over time, particularly in the area of prokaryotic models. This will lead to shaping of an already well-defined family of enzymes, however adding insights and interpretation. While all members generally follow the common ALDH architecture, it is now the smaller, more niche aspects which help us develop the key understanding of these enzymes, ultimately to pick apart ALDH structure, function and disease models in a profound manner.

\section{AUTHOR CONTRIBUTIONS}

KS AD EM, and TS contributed to planning, conception and design of the article, KS AD EM, and TS contributed to idea generation, $\mathrm{KS} \mathrm{AD}$, and TS wrote the review paper, KS and $\mathrm{AD}$ generated the figures, KS constructed tables. KS AD EM, and TS reviewed and revised final version of the paper for publication.

\section{FUNDING}

The authors acknowledge funding from the European Union's Horizon 2020 research and innovation program, Oyster (Open characterisation and modeling environment to drive innovation in advanced nano-architectured and bio-inspired hard/soft interfaces) under grant agreement No. 760827. This funders role in this paper is funding for a research $\mathrm{PhD}$ position.

\section{ACKNOWLEDGMENTS}

The authors acknowledge funding from the European Union's Horizon 2020 research and innovation program, Oyster (Open characterisation and modeling environment to drive innovation in advanced nano-architectured and bio-inspired hard/soft interfaces) under grant agreement No. 760827. The authors acknowledge PyMOL, version 0.99rc6; DeLano Scientific LLC: Palo Alto, CA, United States, 2006 (DeLano Scientific LlC, 2006: Palo Alto) and Pymol 2.0.6 (https://pymol.org/2/), for use in figure generation and visualisation. This review article is dedicated to the memory of Dr Kevin Hayes, 1991 - 2017. 


\section{REFERENCE}

Brocker, C., Vasiliou, M., Carpenter, S., Carpenter, C., Zhang, Y., Wang, X., et al. (2013). Aldehyde Dehydrogenase (ALDH) Superfamily in Plants: Gene Nomenclature and Comparative Genomics. Planta 237, 189-210. doi:10. 1007/s00425-012-1749-0

Chang, J. S., Hsiao, J.-R., and Chen, C.-H. (2017). ALDH2 Polymorphism and Alcohol-Related Cancers in Asians: a Public Health Perspective. J. Biomed. Sci. 24, 1-10. doi:10.1186/s12929-017-0327-y

Chao, Y.-C., Liou, S.-R., Chung, Y.-Y., Tang, H.-S., Hsu, C.-T., Li, T.-K., et al. (1994). Polymorphism of Alcohol and Aldehyde Dehydrogenase Genes and Alcoholic Cirrhosis in Chinese Patients. Hepatology 19, 360-366. doi:10.1002/ hep. 1840190214

Chen, C.-H., Budas, G. R., Churchill, E. N., Disatnik, M.-H., Hurley, T. D., and Mochly-Rosen, D. (2008). Activation of Aldehyde Dehydrogenase-2 Reduces Ischemic Damage to the Heart. Science 321, 1493-1495. doi:10.1126/science. 1158554

Chen, C.-H., Ferreira, J. C. B., Gross, E. R., and Mochly-Rosen, D. (2014). Targeting Aldehyde Dehydrogenase 2: New Therapeutic Opportunities. Physiol. Rev. 94, 1-34. doi:10.1152/physrev.00017.2013

Chen, C.-H., Ferreira, J. C. B., and Mochly-Rosen, D. (2019). ALDH2 and Cardiovascular Disease. In: Aldehyde Dehydrogenases. Singapore: Springer, 53-67. doi:10.1007/978-981-13-6260-6_3

Chen, C., Joo, J. C., Brown, G., Stolnikova, E., Halavaty, A. S., Savchenko, A., et al. (2014). Structure-based Mutational Studies of Substrate Inhibition of Betaine Aldehyde Dehydrogenase BetB from Staphylococcus aureus. Appl. Environ. Microbiol. 80, 3992-4002. doi:10.1128/aem.00215-14

Cho, K. H., Shim, S. H., and Kim, M. (2018). Clinical, Biochemical, and Genetic Aspects of Sjögren-Larsson Syndrome. Clin. Genet. 93, 721-730. doi:10.1111/ cge. 13058

Choudhary, S., Xiao, T., Vergara, L. A., Srivastava, S., Nees, D., Piatigorsky, J., et al. (2005). Role of Aldehyde Dehydrogenase Isozymes in the Defense of Rat Lens and Human Lens Epithelial Cells against Oxidative Stress. Invest. Ophthalmol. Vis. Sci. 46, 259-267. doi:10.1167/iovs.04-0120

Coughlin, C. R., Swanson, M. A., Spector, E., Meeks, N. J., Kronquist, K. E., Aslamy, M., et al. (2018). The Genotypic Spectrum of ALDH7A1 Mutations Resulting in Pyridoxine Dependent Epilepsy: a Common Epileptic Encephalopathy. J. Inherit. Metab. Dis., 1-9. doi:10.1002/jimd.12045

Crabo, A. G., Singh, B., Nguyen, T., Emami, S., Gassner, G. T., and Sazinsky, M. H. (2017). Structure and Biochemistry of Phenylacetaldehyde Dehydrogenase from the Pseudomonas Putida S12 Styrene Catabolic Pathway. Arch. Biochem. Biophys. 616, 47-58. doi:10.1016/j.abb.2017.01.011

D’ambrosio, K., Pailot, A., Talfournier, F., Didierjean, C., Benedetti, E., Aubry, A., et al. (2006). The First Crystal Structure of a Thioacylenzyme Intermediate in the ALDH Family: New Coenzyme Conformation and Relevance to Catalysis. Biochemistry 45, 2978-2986. doi:10.1021/bi0515117

Dayhoff, M. (1976). The Origin and Evolution of Protein Superfamilies. Fed Proc. $35,2132-2138$.

Deak, K. L., Dickerson, M. E., Linney, E., Enterline, D. S., George, T. M., Melvin, E. C., et al. (2005). Analysis ofALDH1A2,CYP26A1,CYP26B1,CRABP1, andCRABP2 in Human Neural Tube Defects Suggests a Possible Association with Alleles inALDH1A2. Birth Defect Res. A. 73, 868-875. doi:10.1002/bdra.20183

Delano Scientific Llc (2006). PyMOL, Version 0.99rc6. C., USA: Palo Alto.

Dupé, V., Matt, N., Garnier, J.-M., Chambon, P., Mark, M., and Ghyselinck, N. B. (2003). A Newborn Lethal Defect Due to Inactivation of Retinaldehyde Dehydrogenase Type 3 Is Prevented by Maternal Retinoic Acid Treatment. Proc. Natl. Acad. Sci. 100, 14036-14041. doi:10.1073/pnas.2336223100

Esterbauer, H., Schaur, R. J., and Zollner, H. (1991). Chemistry and Biochemistry of 4-hydroxynonenal, Malonaldehyde and Related Aldehydes. Free Radic. Biol. Med. 11, 81-128. doi:10.1016/0891-5849(91)90192-6

Estey, T., Cantore, M., Weston, P. A., Carpenter, J. F., Petrash, J. M., and Vasiliou, V. (2007). Mechanisms Involved in the Protection of UV-Induced Protein Inactivation by the Corneal Crystallin ALDH3A1. J. Biol. Chem. 282, 4382-4392. doi:10.1074/jbc.m607546200

Farres, J., Wang, T. T. Y., Cunningham, S. J., and Weiner, H. (1995). Investigation of the Active Site Cysteine Residue of Rat Liver Mitochondrial Aldehyde
Dehydrogenase by Site-Directed Mutagenesis. Biochemistry 34, 2592-2598. doi:10.1021/bi00008a025

Farrés, J., Wang, X., Takahashi, K., Cunningham, S. J., Wang, T. T., and Weiner, H. (1994). Effects of Changing Glutamate 487 to Lysine in Rat and Human Liver Mitochondrial Aldehyde Dehydrogenase. A Model to Study Human (Oriental Type) Class 2 Aldehyde Dehydrogenase. J. Biol. Chem. 269, 13854-13860. doi:10.1016/s0021-9258(17)36725-x

Feldman, R. I., and Weiner, H. (1972). Horse Liver Aldehyde Dehydrogenase. J. Biol. Chem. 247, 267-272. doi:10.1016/s0021-9258(19)45785-2

González-Segura, L., Rudiño-Piñera, E., Muñoz-Clares, R. A., and Horjales, E. (2009). The Crystal Structure of A Ternary Complex of Betaine Aldehyde Dehydrogenase from Pseudomonas aeruginosa Provides New Insight into the Reaction Mechanism and Shows A Novel Binding Mode of the $2^{\prime}$-Phosphate of NADP+ and A Novel Cation Binding Site. J. Mol. Biol. 385, 542-557. doi:10. 1016/j.jmb.2008.10.082

Guo, Y., Tan, L.-J., Lei, S.-F., Yang, T.-L., Chen, X.-D., Zhang, F., et al. (2010). Genome-wide Association Study Identifies ALDH7A1 as a Novel Susceptibility Gene for Osteoporosis. Plos Genet. 6, e1000806. doi:10.1371/journal.pgen. 1000806

Hayes, K., Noor, M., Djeghader, A., Armshaw, P., Pembroke, T., Tofail, S., et al. (2018). The Quaternary Structure of Thermus Thermophilus Aldehyde Dehydrogenase Is Stabilized by an Evolutionary Distinct C-Terminal Arm Extension. Scientific Rep. 8, 1-14. doi:10.1038/s41598-018-31724-8

Hempel, J., Nicholas, H., and Lindahl, R. (1993). Aldehyde Dehydrogenases: Widespread Structural and Functional Diversity within a Shared Framework. Protein Sci. 2, 1890-1900. doi:10.1002/pro.5560021111

Ho, K. K., and Weiner, H. (2005). Isolation and Characterization of an Aldehyde Dehydrogenase Encoded by the aldB Gene of Escherichia coli. $J b$ 187, 1067-1073. doi:10.1128/jb.187.3.1067-1073.2005

Hol, W. G. J., Van Duijnen, P. T., and Berendsen, H. J. C. (1978). The $\alpha$-helix Dipole and the Properties of Proteins. Nature 273, 443-446. doi:10.1038/ 273443a0

Hoog, S. S., Pawlowski, J. E., Alzari, P. M., Penning, T. M., and Lewis, M. (1994). Three-dimensional Structure of Rat Liver 3 Alpha-Hydroxysteroid/dihydrodiol Dehydrogenase: a Member of the Aldo-Keto Reductase Superfamily. Proc. Natl. Acad. Sci. 91, 2517-2521. doi:10.1073/pnas.91.7.2517

Hou, Q., and Bartels, D. (2015). Comparative Study of the Aldehyde Dehydrogenase (ALDH) Gene Superfamily in the Glycophyte Arabidopsis thaliana and Eutrema Halophytes. Ann. Bot. 115, 465-479. doi:10.1093/aob/ mcu152

Hui, P., Nakayama, T., Morita, A., Sato, N., Hishiki, M., Saito, K., et al. (2007). Common Single Nucleotide Polymorphisms in Japanese Patients with Essential Hypertension: Aldehyde Dehydrogenase 2 Gene as a Risk Factor Independent of Alcohol Consumption. Hypertens. Res. 30, 585-592. doi:10.1291/hypres. 30.585

Hurley, T. D., Steinmetz, C. G., and Weiner, H. (1999). "Three-dimensional Structure of Mitochondrial Aldehyde Dehydrogenase," in Enzymology and Molecular Biology of Carbonyl Metabolism 7 (Springer), 15-25. doi:10.1007/ 978-1-4615-4735-8 3

Husemoen, L. L., Fenger, M., Friedrich, N., Tolstrup, J. S., Beenfeldt Fredriksen, S., and Linneberg, A. (2008). The Association of ADH and ALDH Gene Variants with Alcohol Drinking Habits and Cardiovascular Disease Risk Factors. Alcohol. Clin. Exp. Res. 32, 1984-1991. doi:10.1111/j.1530-0277.2008.00780.x

Inagaki, E., Ohshima, N., Takahashi, H., Kuroishi, C., Yokoyama, S., and Tahirov, T. (2006). Crystal Structure of Thermus Thermophilus $\Delta 1$-Pyrroline-5carboxylate Dehydrogenase. J. Mol. Biol. 362, 490-501. doi:10.1016/j.jmb. 2006.07.048

Jackson, B., Brocker, C., Thompson, D. C., Black, W., Vasiliou, K., Nebert, D. W., et al. (2011). Update on the Aldehyde Dehydrogenase Gene (ALDH) Superfamily. Hum. genomics 5, 283. doi:10.1186/1479-7364-5-4-283

Jo, S. A., Kim, E.-K., Park, M. H., Han, C., Park, H.-Y., Jang, Y., et al. (2007). A Glu487Lys Polymorphism in the Gene for Mitochondrial Aldehyde Dehydrogenase 2 Is Associated with Myocardial Infarction in Elderly Korean Men. Clinica Chim. Acta 382, 43-47. doi:10.1016/j.cca.2007.03.016

Kamino, K., Nagasaka, K., Imagawa, M., Yamamoto, H., Yoneda, H., Ueki, A., et al. (2000). Deficiency in Mitochondrial Aldehyde Dehydrogenase Increases the Risk for Late-Onset Alzheimer's Disease in the Japanese Population. Biochem. biophysical Res. Commun. 273, 192-196. doi:10.1006/bbrc.2000.2923 
Kawata, T., Masuda, K., and Yoshino, K. (1975). Presence of Fine Spirals (Spirosomes) inLactobacillus fermentiandLactobacil Lus Casei. Jpn. J. Microbiol. 19, 225-227. doi:10.1111/j.1348-0421.1975.tb00872.x

Keller, M. A., Zander, U., Fuchs, J. E., Kreutz, C., Watschinger, K., Mueller, T., et al. (2014). A Gatekeeper Helix Determines the Substrate Specificity of Sjögren-Larsson Syndrome Enzyme Fatty Aldehyde Dehydrogenase. Nat. Commun. 5, 1-12. doi:10.1038/ncomms5439

Kelson, T. L., Secor McVoy, J. R., and Rizzo, W. B. (1997). Human Liver Fatty Aldehyde Dehydrogenase: Microsomal Localization, Purification, and Biochemical Characterization. Biochim. Biophys. Acta (Bba) - Gen. Subjects 1335, 99-110. doi:10.1016/s0304-4165(96)00126-2

Kessler, D., Leibrecht, I., and Knappe, J. (1991). Pyruvate-formate-lyase-deactivase and Acetyl-CoA Reductase Activities ofEscherichia Colireside on a Polymeric Protein Particle Encoded byadhE. FEBS Lett. 281, 59-63. doi:10.1016/00145793(91)80358-a

Kim, G., Azmi, L., Jang, S., Jung, T., Hebert, H., Roe, A. J., et al. (2019). Aldehydealcohol Dehydrogenase Forms a High-Order Spirosome Architecture Critical for its Activity. Nat. Commun. 10, 1-11. doi:10.1038/s41467-019-12427-8

Kim, G., Yang, J., Jang, J., Choi, J.-S., Roe, A. J., Byron, O., et al. (2020). Aldehydealcohol Dehydrogenase Undergoes Structural Transition to Form Extended Spirosomes for Substrate Channeling. Commun. Biol. 3, 1-9. doi:10.1038/ s42003-020-1030-1

Kim, J.-I., Ganesan, S., Luo, S. X., Wu, Y.-W., Park, E., Huang, E. J., et al. (2015). Aldehyde Dehydrogenase lal Mediates a GABA Synthesis Pathway in Midbrain Dopaminergic Neurons. Science 350, 102-106. doi:10.1126/science. aac 4690

Klyosov, A. A. (1996). Kinetics and Specificity of Human Liver Aldehyde Dehydrogenases toward Aliphatic, Aromatic, and Fused Polycyclic Aldehydes†. Biochemistry 35, 4457-4467. doi:10.1021/bi9521102

Koppaka, V., Thompson, D. C., Chen, Y., Ellermann, M., Nicolaou, K. C., Juvonen, R. O., et al. (2012). Aldehyde Dehydrogenase Inhibitors: a Comprehensive Review of the Pharmacology, Mechanism of Action, Substrate Specificity, and Clinical Application. Pharmacol. Rev. 64, 520-539. doi:10.1124/pr.111.005538

Korasick, D. A., Tanner, J. J., and Henzl, M. T. (2017). Impact of Disease-Linked Mutations Targeting the Oligomerization Interfaces of Aldehyde Dehydrogenase 7A1. Chemico-biological interactions 276, 31-39. doi:10. 1016/j.cbi.2017.01.002

Laciak, A. R., Korasick, D. A., Gates, K. S., and Tanner, J. J. (2020). Structural Analysis of Pathogenic Mutations Targeting Glu427 of ALDH7A1, the Hot Spot Residue of Pyridoxine-dependent Epilepsy. Jrnl Inher Metab. Disea 43, 635-644. doi:10.1002/jimd.12184

Larson, H. N., Weiner, H., and Hurley, T. D. (2005). Disruption of the Coenzyme Binding Site and Dimer Interface Revealed in the Crystal Structure of Mitochondrial Aldehyde Dehydrogenase "Asian" Variant. J. Biol. Chem. 280, 30550-30556. doi:10.1074/jbc.m502345200

Larson, H. N., Zhou, J., Chen, Z., Stamler, J. S., Weiner, H., and Hurley, T. D. (2007). Structural and Functional Consequences of Coenzyme Binding to the Inactive Asian Variant of Mitochondrial Aldehyde Dehydrogenase. J. Biol. Chem. 282, 12940-12950. doi:10.1074/jbc.m607959200

Lassen, N., Bateman, J. B., Estey, T., Kuszak, J. R., Nees, D. W., Piatigorsky, J., et al. (2007). Multiple and Additive Functions of ALDH3A1 and ALDH1A1. J. Biol. Chem. 282, 25668-25676. doi:10.1074/jbc.m702076200

Lassen, N., Black, W. J., Estey, T., and Vasiliou, V. (2008). The Role of Corneal Crystallins in the Cellular Defense Mechanisms against Oxidative Stress. Semin. Cel. Dev. Biol. 19, 100-112. doi:10.1016/j.semcdb.2007.10.004

Lindahl, R. (1992). Aldehyde Dehydrogenases and Their Role in Carcinogenesis. Crit. Rev. Biochem. Mol. Biol. 27, 283-335. doi:10.3109/10409239209082565

Lindahl, R., and Hempel, J. (1990). "Aldehyde Dehydrogenases: What Can Be Learned from a Baker's Dozen Sequences?," in Enzymology and Molecular Biology of Carbonyl Metabolism 3 (New York, NY: Springer), 1-8. doi:10.1007/ 978-1-4684-5901-2_1

Liu, L.-K., and Tanner, J. J. (2019). Crystal Structure of Aldehyde Dehydrogenase 16 Reveals Trans-hierarchical Structural Similarity and a New Dimer. J. Mol. Biol. 431, 524-541. doi:10.1016/j.jmb.2018.11.030

Liu, Z.-J., Sun, Y.-J., Rose, J., Chung, Y.-J., Hsiao, C.-D., Chang, W.-R., et al. (1997). The First Structure of an Aldehyde Dehydrogenase Reveals Novel Interactions between NAD and the Rossmann Fold. Nat. Struct. Mol. Biol. 4, 317-326. doi:10.1038/nsb0497-317
Luo, M., Singh, R. K., and Tanner, J. J. (2013). Structural Determinants of Oligomerization of $\Delta 1$-Pyrroline-5-Carboxylate Dehydrogenase: Identification of a Hexamerization Hot Spot. J. Mol. Biol. 425, 3106-3120. doi:10.1016/j.jmb.2013.05.027

Marchitti, S. A., Brocker, C., Stagos, D., and Vasiliou, V. (2008). Non-P450 Aldehyde Oxidizing Enzymes: the Aldehyde Dehydrogenase Superfamily. Expert Opin. Drug Metab. Toxicol. 4, 697-720. doi:10.1517/17425255.4.6.697

Marchitti, S. A., Chen, Y., Thompson, D. C., and Vasiliou, V. (2011). Ultraviolet Radiation: Cellular Antioxidant Response and the Role of Ocular Aldehyde Dehydrogenase Enzymes. Eye \& contact lens 37, 206-213. doi:10.1097/icl. ob013e3182212642

Marchitti, S. A., Deitrich, R. A., and Vasiliou, V. (2007). Neurotoxicity and Metabolism of the Catecholamine-Derived 3,4-Dihydroxyphenylacetaldehyde and 3,4-Dihydroxyphenylglycolaldehyde: The Role of Aldehyde Dehydrogenase. Pharmacol. Rev. 59, 125-150. doi:10.1124/pr.59.2.1

Mellema, S., Eichenberger, W., Rawyler, A., Suter, M., Tadege, M., and Kuhlemeier, C. (2002). The Ethanolic Fermentation Pathway Supports Respiration and Lipid Biosynthesis in Tobacco Pollen. Plant J. 30, 329-336. doi:10.1046/j.1365313x.2002.01293.x

Mills, P. B., Footitt, E. J., Mills, K. A., Tuschl, K., Aylett, S., Varadkar, S., et al. (2010). Genotypic and Phenotypic Spectrum of Pyridoxine-dependent Epilepsy (ALDH7A1 Deficiency). Brain 133, 2148-2159. doi:10.1093/brain/awq143

Mills, P. B., Struys, E., Jakobs, C., Plecko, B., Baxter, P., Baumgartner, M., et al. (2006). Mutations in Antiquitin in Individuals with Pyridoxine-dependent Seizures. Nat. Med. 12, 307-309. doi:10.1038/nm1366

Moore, S. A., Baker, H. M., Blythe, T. J., Kitson, K. E., Kitson, T. M., and Baker, E. N. (1998). Sheep Liver Cytosolic Aldehyde Dehydrogenase: the Structure Reveals the Basis for the Retinal Specificity of Class 1 Aldehyde Dehydrogenases. Structure 6, 1541-1551. doi:10.1016/s0969-2126(98)00152-x

Moreb, J. (2008). Aldehyde Dehydrogenase as a Marker for Stem Cells. Cur Stem Cell Res Ther.scr 3, 237-246. doi:10.2174/157488808786734006

Moretti, A., Li, J., Donini, S., Sobol, R. W., Rizzi, M., and Garavaglia, S. (2016). Crystal Structure of Human Aldehyde Dehydrogenase 1A3 Complexed with NAD+ and Retinoic Acid. Scientific Rep. 6, 1-12. doi:10.1038/srep35710

Muzio, G., Maggiora, M., Paiuzzi, E., Oraldi, M., and Canuto, R. A. (2012). Aldehyde Dehydrogenases and Cell Proliferation. Free Radic. Biol. Med. 52, 735-746. doi:10.1016/j.freeradbiomed.2011.11.033

Ouyang, Y., Li, Q., Kuang, X., Wang, H., Wu, J., Ayepa, E., et al. (2020). YMR152W from Saccharomyces cerevisiae Encoding a Novel Aldehyde Reductase for Detoxification of Aldehydes Derived from Lignocellulosic Biomass. J. Biosci. Bioeng. 131, 39-46. doi:10.1016/j.jbiosc.2020.09.004

Palmfeldt, J., Vang, S., Stenbroen, V., Pavlou, E., Baycheva, M., Buchal, G., et al. (2011). Proteomics Reveals that Redox Regulation Is Disrupted in Patients with Ethylmalonic Encephalopathy. J. Proteome Res. 10, 2389-2396. doi:10.1021/pr101218d

Palo, O. M., Soronen, P., Silander, K., Varilo, T., Tuononen, K., Kieseppä, T., et al. (2010). Identification of Susceptibility Loci at $7 \mathrm{q} 31$ and $9 \mathrm{p} 13$ for Bipolar Disorder in an Isolated Population. Am. J. Med. Genet. B Neuropsychiatr. Genet. 153B, 723-735. doi:10.1002/ajmg.b.31039

Pavan, M., Ruiz, V. F., Silva, F. A., Sobreira, T. J., Cravo, R. M., Vasconcelos, M., et al. (2009). ALDH1A2 (RALDH2) Genetic Variation in Human Congenital Heart Disease. BMC Med. Genet. 10, 113. doi:10.1186/1471-2350-10-113

Pemberton, T. A., Srivastava, D., Sanyal, N., Henzl, M. T., Becker, D. F., and Tanner, J. J. (2014). Structural Studies of Yeast $\Delta 1$-Pyrroline-5-carboxylate Dehydrogenase (ALDH4A1): Active Site Flexibility and Oligomeric State. Biochemistry 53, 1350-1359. doi:10.1021/bi500048b

Pequerul, R., Vera, J., Giménez-Dejoz, J., Crespo, I., Coines, J., Porté, S., et al. (2020). Structural and Kinetic Features of Aldehyde Dehydrogenase 1A (ALDH1A) Subfamily Members, Cancer Stem Cell Markers Active in Retinoic Acid Biosynthesis. Arch. Biochem. Biophys. 681, 108256. doi:10. 1016/j.abb.2020.108256

Perez-Miller, S., Younus, H., Vanam, R., Chen, C.-H., Mochly-Rosen, D., and Hurley, T. D. (2010). Alda-1 Is an Agonist and Chemical Chaperone for the Common Human Aldehyde Dehydrogenase 2 Variant. Nat. Struct. Mol. Biol. 17, 159-164. doi:10.1038/nsmb.1737

Perozich, J., Nicholas, H., Wang, B. C., Lindahl, R., and Hempel, J. (1999). Relationships within the Aldehyde Dehydrogenase Extended Family. Protein Sci. 8, 137-146. doi:10.1110/ps.8.1.137

Perozich, J., Kuo, I., Wang, B. C., Boesch, J. S., Lindahl, R., and Hempel, J. (2000). Shifting the NAD/NADP Preference in Class 3 Aldehyde 
Dehydrogenase. Eur. J. Biochem. 267, 6197-6203. doi:10.1046/j.1432-1327. 2000.01697.x

Perozich, J., Nicholas, H., Lindahl, R., and Hempel, J. (1999). "The Big Book of Aldehyde Dehydrogenase Sequences," in Enzymology and Molecular Biology of Carbonyl Metabolism 7 (New York, NY: Springer), 1-7. doi:10.1007/978-14615-4735-8_1

Piatigorsky, J. (1998). Multifunctional Lens Crystallins and Corneal Enzymes: More Than Meets the Eye. Ann. NY Acad Sci 842, 7-15. doi:10.1111/j.17496632.1998.tb09626.x

Plecko, B., Paul, K., Paschke, E., Stoeckler-Ipsiroglu, S., Struys, E., Jakobs, C., et al. (2007). Biochemical and Molecular Characterization of 18 Patients with Pyridoxine-dependent Epilepsy and Mutations of the Antiquitin (ALDH7A1) Gene. Hum. Mutat. 28, 19-26. doi:10.1002/humu.20433

Pony, P., Rapisarda, C., Terradot, L., Marza, E., and Fronzes, R. (2020). Filamentation of the Bacterial Bi-functional Alcohol/aldehyde Dehydrogenase AdhE Is Essential for Substrate Channeling and Enzymatic Regulation. Nat. Commun. 11, 1-11. doi:10.1038/s41467-020-15214-y

Riveros-Rosas, H., González-Segura, L., Julián-Sánchez, A., Díaz-Sánchez, Á. G., and Muñoz-Clares, R. A. (2013). Structural Determinants of Substrate Specificity in Aldehyde Dehydrogenases. Chemico-biological interactions 202, 51-61. doi:10.1016/j.cbi.2012.11.015

Riveros-Rosas, H., Julián-Sánchez, A., Moreno-Hagelsieb, G., and Muñoz-Clares, R. A. (2019). Aldehyde Dehydrogenase Diversity in Bacteria of the Pseudomonas Genus. Chemico-biological interactions 304, 83-87. doi:10. 1016/j.cbi.2019.03.006

Rodríguez-Zavala, J. S., Allali-Hassani, A., and Weiner, H. (2006). Characterization of E. coli Tetrameric Aldehyde Dehydrogenases with Atypical Properties Compared to Other Aldehyde Dehydrogenases. Protein Sci. 15, 1387-1396. doi:10.1110/ps.052039606

Rodriguez-Zavala, J., and Weiner, H. (2001). Role of the C-Terminal Tail on the Quaternary Structure of Aldehyde Dehydrogenases. Chemico-Biological Interactions 130-132, 151-160. doi:10.1016/s0009-2797(00)00230-1

Rossmann, M. G., Moras, D., and Olsen, K. W. (1974). Chemical and Biological Evolution of a Nucleotide-Binding Protein. Nature 250, 194-199. doi:10.1038/ 250194a0

Sass, J. O., Walter, M., Shield, J. P. H., Atherton, A. M., Garg, U., Scott, D., et al. (2012). 3-Hydroxyisobutyrate Aciduria and Mutations in the ALDH6A1 Gene Coding for Methylmalonate Semialdehyde Dehydrogenase. J. Inherit. Metab. Dis. 35, 437-442. doi:10.1007/s10545-011-9381-x

Scharer, G., Brocker, C., Vasiliou, V., Creadon-Swindell, G., Gallagher, R. C., Spector, E., et al. (2010). The Genotypic and Phenotypic Spectrum of Pyridoxine-dependent Epilepsy Due to Mutations in ALDH7A1. J. Inherit. Metab. Dis. 33, 571-581. doi:10.1007/s10545-010-9187-2

Seitz, H. K., and Stickel, F. (2010). Acetaldehyde as an Underestimated Risk Factor for Cancer Development: Role of Genetics in Ethanol Metabolism. Genes Nutr. 5, 121-128. doi:10.1007/s12263-009-0154-1

Sharkey, M. A., Oliveira, T. F., Engel, P. C., and Khan, A. R. (2013). Structure of NADP+-dependent Glutamate Dehydrogenase fromEscherichia Coli Reflections on the Basis of Coenzyme Specificity in the Family of Glutamate Dehydrogenases. Febs J. 280, 4681-4692. doi:10.1111/febs.12439

Sheikh, S., Ni, L., Hurley, T. D., and Weiner, H. (1997). The Potential Roles of the Conserved Amino Acids in Human Liver Mitochondrial Aldehyde Dehydrogenase. J. Biol. Chem. 272, 18817-18822. doi:10.1074/jbc.272.30.18817

Shin, J.-H., Kim, S.-R., and An, G. (2009). Rice Aldehyde Dehydrogenase7 Is Needed for Seed Maturation and Viability. Plant Physiol. 149, 905-915. doi:10. 1104/pp.108.130716

Singh, S., Brocker, C., Koppaka, V., Chen, Y., Jackson, B. C., Matsumoto, A., et al. (2013). Aldehyde Dehydrogenases in Cellular Responses to Oxidative/ electrophilicstress. Free Radic. Biol. Med. 56, 89-101. doi:10.1016/j. freeradbiomed.2012.11.010

Sládek, N. E. (2003). Human Aldehyde Dehydrogenases: Potential Pathological, Pharmacological, and Toxicological Impact. J. Biochem. Mol. Toxicol. 17, 7-23. doi:10.1002/jbt.10057

Sobreira, T. J. P., Marlétaz, F., Simões-Costa, M., Schechtman, D., Pereira, A. C., Brunet, F., et al. (2011). Structural Shifts of Aldehyde Dehydrogenase Enzymes Were Instrumental for the Early Evolution of Retinoid-dependent Axial Patterning in Metazoans. Proc. Natl. Acad. Sci. USA 108, 226-231. doi:10. 1073/pnas.1011223108
Steinmetz, C. G., Xie, P., Weiner, H., and Hurley, T. D. (1997). Structure of Mitochondrial Aldehyde Dehydrogenase: the Genetic Component of Ethanol Aversion. Structure 5, 701-711. doi:10.1016/s0969-2126(97)00224-4

Stenson, P. D., Ball, E., Howells, K., Phillips, A., Mort, M., and Cooper, D. N. (2008). Human Gene Mutation Database: towards a Comprehensive Central Mutation Database. J. Med. Genet. 45, 124-126. doi:10.1136/jmg.2007.055210

Sulem, P., Gudbjartsson, D. F., Walters, G. B., Helgadottir, H. T., Helgason, A., Gudjonsson, S. A., et al. (2011). Identification of Low-Frequency Variants Associated with Gout and Serum Uric Acid Levels. Nat. Genet. 43, 1127-1130. doi:10.1038/ng.972

Sydow, K., Daiber, A., Oelze, M., Chen, Z., August, M., Wendt, M., et al. (2004). Central Role of Mitochondrial Aldehyde Dehydrogenase and Reactive Oxygen Species in Nitroglycerin Tolerance and Cross-Tolerance. J. Clin. Invest. 113, 482-489. doi:10.1172/jci200419267

Tao, X., Zhang, Z., Zhang, X., Li, H., Sun, H., Mao, Z.-W., et al. (2020). Structural Insight into the Substrate Gating Mechanism by Staphylococcus aureus Aldehyde Dehydrogenase. CCS Chem. 2, 946-954. doi:10.31635/ccschem. 020.202000219

Van Karnebeek, C. D. M., Tiebout, S. A., Niermeijer, J., Poll-The, B. T., Ghani, A., Coughlin, C. R., et al. (2016). Pyridoxine-dependent Epilepsy: an Expanding Clinical Spectrum. Pediatr. Neurol. 59, 6-12. doi:10.1016/j.pediatrneurol.2015.12.013

Vasiliou, V., Bairoch, A., Tipton, K. F., and Nebert, D. W. (1999). Eukaryotic Aldehyde Dehydrogenase (ALDH) Genes: Human Polymorphisms, and Recommended Nomenclature Based on Divergent Evolution and Chromosomal Mapping. Pharmacogenetics and Genomics 9, 421-434. doi:10.1097/00008571-199910000-00004

Vasiliou, V., and Nebert, D. W. (2005). Analysis and Update of the Human Aldehyde Dehydrogenase (ALDH) Gene Family. Hum. genomics 2, 1-6. doi:10. 1186/1479-7364-2-2-138

Vasiliou, V., Thompson, D. C., Smith, C., Fujita, M., and Chen, Y. (2013). Aldehyde Dehydrogenases: from Eye Crystallins to Metabolic Disease and Cancer Stem Cells. Chemico-biological interactions 202, 2-10. doi:10.1016/j. cbi.2012.10.026

Vassalli, G. (2019). Aldehyde Dehydrogenases: Not Just Markers, but Functional Regulators of Stem Cells. Stem Cell Int. 2019, 3904645. doi:10.1155/2019/ 3904645

Voulgaridou, G.-P., Tsochantaridis, I., Mantso, T., Franco, R., Panayiotidis, M. I., and Pappa, A. (2017). Human Aldehyde Dehydrogenase 3A1 (ALDH3A1) Exhibits Chaperone-like Function. Int. J. Biochem. Cel Biol. 89, 16-24. doi:10. 1016/j.biocel.2017.05.017

Voulgaridou, G.-P., Tsochantaridis, I., Tolkas, C., Franco, R., Giatromanolaki, A., Panayiotidis, M. I., et al. (2020). Aldehyde Dehydrogenase 3A1 Confers Oxidative Stress Resistance Accompanied by Altered DNA Damage Response in Human Corneal Epithelial Cells. Free Radic. Biol. Med. 150, 66-74. doi:10.1016/j.freeradbiomed.2020.01.183

Wierenga, R. K., and Hol, W. G. J. (1983). Predicted Nucleotide-Binding Properties of P21 Protein and its Cancer-Associated Variant. Nature 302, 842-844. doi:10.1038/302842a0

Wierenga, R. K., Terpstra, P., and Hol, W. G. J. (1986). Prediction of the Occurrence of the ADP-Binding $\beta a \beta$-fold in Proteins, Using an Amino Acid Sequence Fingerprint. J. Mol. Biol. 187, 101-107. doi:10.1016/0022-2836(86)90409-2

Wilson, D., Bohren, K., Gabbay, K., and Quiocho, F. (1992). An Unlikely Sugar Substrate Site in the 1.65 A Structure of the Human Aldose Reductase Holoenzyme Implicated in Diabetic Complications. Science 257, 81-84. doi:10.1126/science.1621098

Yoshida, A., Hsu, L. C., and Davé, V. (1992). Retinal Oxidation Activity and Biological Role of Human Cytosolic Aldehyde Dehydrogenase. Enzyme 46, 239-244. doi:10.1159/000468794

Conflict of Interest: The authors declare that the research was conducted in the absence of any commercial or financial relationships that could be construed as a potential conflict of interest.

Copyright $\odot 2021$ Shortall, Djeghader, Magner and Soulimane. This is an openaccess article distributed under the terms of the Creative Commons Attribution License (CC BY). The use, distribution or reproduction in other forums is permitted, provided the original author(s) and the copyright owner(s) are credited and that the original publication in this journal is cited, in accordance with accepted academic practice. No use, distribution or reproduction is permitted which does not comply with these terms. 